\title{
Enquête Ethnobotanique Sur La Prise En Charge Traditionnelle De L'infertilité Féminine Dans La Région Sanitaire Des Savanes Au Togo
}

Assouma Akim Françoise (Docteur en Pharmacie)

Faculté de Médecine, de Pharmacie et d'Odontostomatologie, Université Cheikh Anta Diop (UCAD) Dakar, Sénégal

Koudouvo Koffi (Maître-Assistant)

Laboratoire de Physiologie et Pharmacologie, Faculté des Sciences (FDS), Université de Lomé (UL), Lomé-Togo / Africa Centre of Excellence in Phytomedicine Research and Development, Faculty of Pharmaceutical

Sciences, University of Jos, Nigeria / World Bank

Diatta William (Maître-Assistant)

Bassene Emmanuel (Professeur Titulaire)

Faculté de Médecine, de Pharmacie et d'Odontostomatologie, UCAD,

Dakar, Sénégal

Tougoma Atèhèzi (Master en Ethnopharmacologie)

Laboratoire de Physiologie et Pharmacologie, FDS, UL, Lomé-Togo

Novidzro Mawuéna Kosi (Maître-Assistant)

Laboratoire des Essences Végétales et des Arômes Naturelles, FDS,

UL, Lomé-Togo

Guelly Atsu Kudzo (Professeur Titulaire)

Laboratoire de Botanique et Ecologie Végétale Appliquée, FDS, UL, Lomé-Togo

Dougnon Jacques (Maître de Conférences)

Laboratoire de Recherche en Biologie Appliquée, Ecole Polytechnique d'Abomey-Calavi, Université d'Abomey Calavi, République du Bénin

Agbonon Amégnona (Professeur Titulaire)

Laboratoire de Physiologie et Pharmacologie, FDS, UL, Lomé-Togo

Tozo Koffi Sodokè (Professeur Titulaire)

Laboratoire de Botanique et Ecologie Végétale Appliquée, FDS, UL,

Lomé-Togo

Gbeassor Messanvi (Professeur Titulaire)

Centre de Recherche et de Formation sur les Plantes Médicinales, FDS, UL, Lomé-Togo 


\begin{abstract}
Introduction: The management of female infertility by the conventional medicine is very expensive and its coast is unavailable for the very poor populations. In Africa, an important recourses of medicinal plants are available for health care. Objective: The present study was led to recorder traditional remedies use to treat female infertility in the Sanitary Savannas Region of Togo. Methodology: For data collection, semi interview was used. Data were processed and analysed using ethnobotanical quantitative index. The Selection by Progressive Elimination (SPE) was essentially used for the choice of species. Results: With 51 practitioners interviewed, 79 recipes constituted by 87 species belonging to 48 botanical families were collected. Combretaceae $(12,64 \%)$ and Rubiaceae $(5,7 \%)$ were the most frequent families. Trees $(36,78 \%)$ and shrubs $(27,58 \%)$ were the main ports. Roots $(29,17 \%)$ and leaves $(27,5 \%)$ were the most used parts. Recipes were prepared frequently by decoction $(51,88 \%)$ and powder $(38,12 \%)$, and were principally taken orally $(47,28 \%)$ and by body bath $(43,48 \%)$. Sexually transmitted infections (STI) (143 citations) and Painful menstruations (PM) (108 citations) were the most frequent pathologies. The highest Informants consensus factors were 0,61 and 0,56 respectively for STI and PM. The most important Fidelity degrees was $25,49 \%$ for STI related to Capsicum frutescens and Piper guineense. Related to Aframomum melegueta, Piper guineense and Zanthoxylum zanthoxyloides the Fidelity degree was 19,61\% for PM and STI. Blighia sapida, Ganoderma colossus, Kigelia africana and Mangifera indica were selected. Conclusion: Plants with highest fidelity degree and those selected by SPE will be subjected to laboratory tests.
\end{abstract}

Keywords: Ethnopharmacology, medicinal plants, female infertility, Savannah Region, Togo

\title{
Résumé
}

Introduction : La prise en charge de l'infertilité féminine en médecine conventionnelle est assez onéreuse et inaccessible aux couches sociales très pauvres. En Afrique, il existe un grand recours aux plantes utilisées pour les soins. Objectif : La présente étude a été menée pour recenser les remèdes traditionnels anti-infertilité féminine, utilisées dans la Région sanitaire des Savanes du Togo. Méthodologie : L'interview semi structurée a été utilisée pour la collecte des données. Les données ont été traitées et analysées par des techniques quantitatives ethnobotaniques. Les espèces retenues ont été essentiellement sélectionnées par élimination progressive (SEP). Résultats : Chez 51 praticiens, 79 recettes constituées par 87 espèces appartenant à 48 
familles, ont été recensées. Les Combretaceae $(12,64 \%)$ et les Rubiaceae $(5,7 \%)$ ont été les familles plus fréquentes. Les Arbres (36,78\%), les arbustes $(27,58 \%)$ et les herbes $(24,14 \%)$ ont été les types biologiques majoritaires. Les Racines $(29,17 \%)$ et les feuilles $(27,5 \%)$ ont été les organes plus utilisés. Les recettes ont été fréquemment préparées par décoction $(51,88 \%)$ et poudre $(38,12 \%)$, et administrées par voie orale $(47,28 \%)$ et par bain corporel (43,48\%). Infections sexuellement transmissibles (IST) (143 citations) et Règles douloureuses (RD) (108 citations) ont été les pathologies les plus fréquentes. Les Facteurs de consensus les plus élevées ont été de 0,61 et 0,56 respectivement pour IST et RD. Les degrés de fidélité les plus importants ont été de 25,49\% pour IST par rapport à Capsicum frutescens et Piper guineense, de $19,61 \%$ pour ITS et RD relativement à Aframomum melegueta, Piper guineense et Zanthoxylum zanthoxyloides. Blighia sapida, Ganoderma colossus, Kigelia africana et Mangifera indica ont été sélectionnées. Conclusion : Les plantes avec des degrés de fidélité élevés et celles sélectionnées par la SEP feront l'objet d'analyses expérimentales.

Mots clés : Ethnopharmacologie, plantes médicinales, infertilité féminine, Région des Savanes, Togo

\section{Introduction}

Les recherches sur l'utilisation des plantes en médecine traditionnelle ont permis la découverte de nombreux principes actifs employés aujourd'hui en médecine moderne (Cragg, et al., 2002, Soh et al., 2007 ; Leonti et al., 2017). Une diversité de médicaments sont des molécules dérivant uniquement des plantes ou en sont des homologues structuraux utilisés pour traiter une multitude de troubles dont les maladies du système reproducteur telles que les fibrome, les myomes, les stérilités féminine et masculine, les prostatites, les infertilités féminine et masculine, les cancers du sein et de l'utérus, les aménorrhées (Sakamoto, et $a l .$, 1988. ; Al-Quarawi, et al., 2000, Leonti et al., 2017).

Les traitements proposés par la médecine moderne pour traiter ces maladies sont souvent très couteux alors que leurs taux de réussite sont très faibles. Les malades surtout ceux des milieux pauvres de l'Afrique subsaharienne qui représentent les couches les plus touchées, sont par conséquent incapables de s'offrir de tels traitements. L'utilisation des plantes médicinales constitue pour ces couches, l'une des alternatives pour résoudre ce problème surtout le cas des infertilités. Les plantes utilisées pour traiter l'infertilité féminine sont peu valorisées dans la sous-région ouest africaine. Au Togo, rares sont les études qui ont répertoriées les espèces végétales de même que les remèdes dans lesquels elles sont utilisées pour traiter l'infertilité féminine (Adjanohoun et al., 1986; Batawila et $a l ., 2007$ ). La présente étude a été 
réalisée pour recenser par interview semi structurée, les plantes et recettes de plantes utilisées en médecine traditionnelle de la Région sanitaire des Savanes du Togo pour soigner l'infertilité féminine. La finalité de l'étude est de sélectionner des plantes et recettes de plantes en vue de futurs tests sur des modèles de laboratoire pour vérifier leurs propriétés pharmacologiques.

\section{Matériel et méthode}

\section{- Zone d'étude}

La Région des Savanes (Figure 1) située au Nord du Togo, entre $0^{\circ}$ et $11^{\circ}$ de longitude Est et $10^{\circ}$ de latitude Nord, est la zone d'étude. Elle est limitée au Nord par le Burkina-Faso, au Sud par la région de la Kara, à 1'Est par le Bénin et à l'Ouest par le Ghana. Elle couvre une superficie de $8470 \mathrm{~km}^{2}$. La population de la Région est estimée au dernier recensement de 2010 au Togo à 828.224 habitants (D.G.S.C.N.T, 2011). Les langues les plus parlées sont le Moba et le Gourma. Le relief est caractérisé par une vaste plaine aux vallées relativement basses et plates qui constituent une véritable cuvette où coulent de nombreuses rivières. Le climat de la Région est de type tropical soudanoguinéen caractérisé par deux saisons. La saison pluvieuse est courte, va de Mai à Septembre, le vent qui y souffle étant la Mousson. La saison sèche d'Octobre à Avril est longue et marquée par le Harmattan et une forte insolation avec des températures variables, très élevées et pouvant atteindre $38^{\circ}$ et $45^{\circ} \mathrm{C}$ entre Novembre à Janvier (M.S., 2015).

\section{- Collecte des données}

Cinquante et un PMT (Praticiens de la médecine traditionnelle) de la Région, constitués de 43 tradithérapeutes et 8 herboristes, définis suivant le ratio 1/10000 habitants (D.G.S.C.N.T, 2011; M.S., 2015), volontaires à l'enquête, ont été interviewés après consentement éclairé. Les leaders traditionnels tels que les Chefs de village et les Responsables des associations de praticiens de la médecine traditionnelle du Togo ont servi de facilitateurs pour la réalisation des enquêtes. L'interview semi-structurée (Koudouvo, K., 2009 ; Koudouvo et al., 2011; Karou et al., 2011; Denou et al., 2016a, 2016b) par application d'un questionnaire, et des réunions en groupe focal (Koudouvo et $a l ., 2011)$ ont été les méthodes de collecte des données. Certaines pathologies de la reproduction humaine chez la femme telles que les fibromes, les myomes, les tumeurs ovariens, la blennorragie, la syphilis et la gonococcie, ont été les pathologies utilisées pour amorcer la notion 'd'infertilité féminine", afin de faciliter l'application du questionnaire. Les espèces et recettes de plantes utilisées dans le traitement de l'infertilité féminine, les noms des espèces en langue locale, les parties utilisées, les modes de préparation, les voies d'administration, les modes d'emploi et d'éventuelles mises en garde ou contre-indications, ont été les caractéristiques 
ethnopharmacologiques recherchées (Koudouvo et al., 2011; Denou et al., 2016a).

\section{- Analyse et traitement des données}

La flore Analytique du Bénin (Akoègninou et al., 2006) et la base de données en ligne de PROTA (Plant Resources of Tropical Africa) sur le site internet www.prota4u.org ont été utilisées pour l'identification botanique des espèces de plantes. Les identifications ont été confirmées par l'équipe du Prof. Koffi AKPAGANA du Togo. La précision des noms en langues locales a été réalisée en référence à Adjanohoun et al., (1986; 1989), au Dictionnaire Monographique de l'Afrique de l'Ouest (Eklu-Natey et al., 2012) et à la Pharmacopée de l'Afrique de l'Ouest (P.A.O, 2013).

Les logiciels EPI Info 10.1 et SPPS 12.0 ont servi pour le traitement statistique des données.

Le Facteur de consensus autour des pathologies pour les espèces recensées (Heinrich et al., 1998, Gazzaneo et al., 2005, Aburjai et al., 2006, Teklehaymanot et $a l ., 2007$ ) a été déterminé par la formule suivante : $\mathrm{FC}=\mathrm{N}$ $\mathrm{Ne} / \mathrm{N}-1$ où $\mathbf{N}$ est le nombre de fois que la pathologie est citée et $\mathbf{N e}$, le nombre total d'espèces citées pour la soigner.

Le Degré de fidélité caractéristique de l'importance de la pathologie parmi les pathologies indiquées (Alexiades, 1996, Teklehaymanot et al., 2007), a été déterminé par la formule :

$\mathrm{DF}=\mathrm{Np} / \mathrm{Nx} 100$ avec $\mathbf{N p}$ désignant le nombre d'enquêtés qui ont cité la plante pour la pathologie et $\mathbf{N}$, le nombre total de personnes enquêtées.

\section{- $\quad$ Sélection de plantes et recettes de plantes}

La Sélection par Elimination Progressive (SEP) (Koudouvo, 2009; Denou et $a l ., 2016$; Koudouvo et $a l ., 2016$ ) prenant en compte les recettes de plantes uniques, a été la technique de sélection de plantes pour de futurs tests de laboratoire. La présente étude a amélioré cette méthode de sélection. La Sélection par Elimination Progressive Améliorée (SEPA) a élargi la SEP aux recettes de deux, de trois et de quatre espèces en vue de choisir non seulement des espèces uniques mais aussi des recettes de plantes associées, pour les tests. Les valeurs du DF ont servi complémentairement à ce choix. 


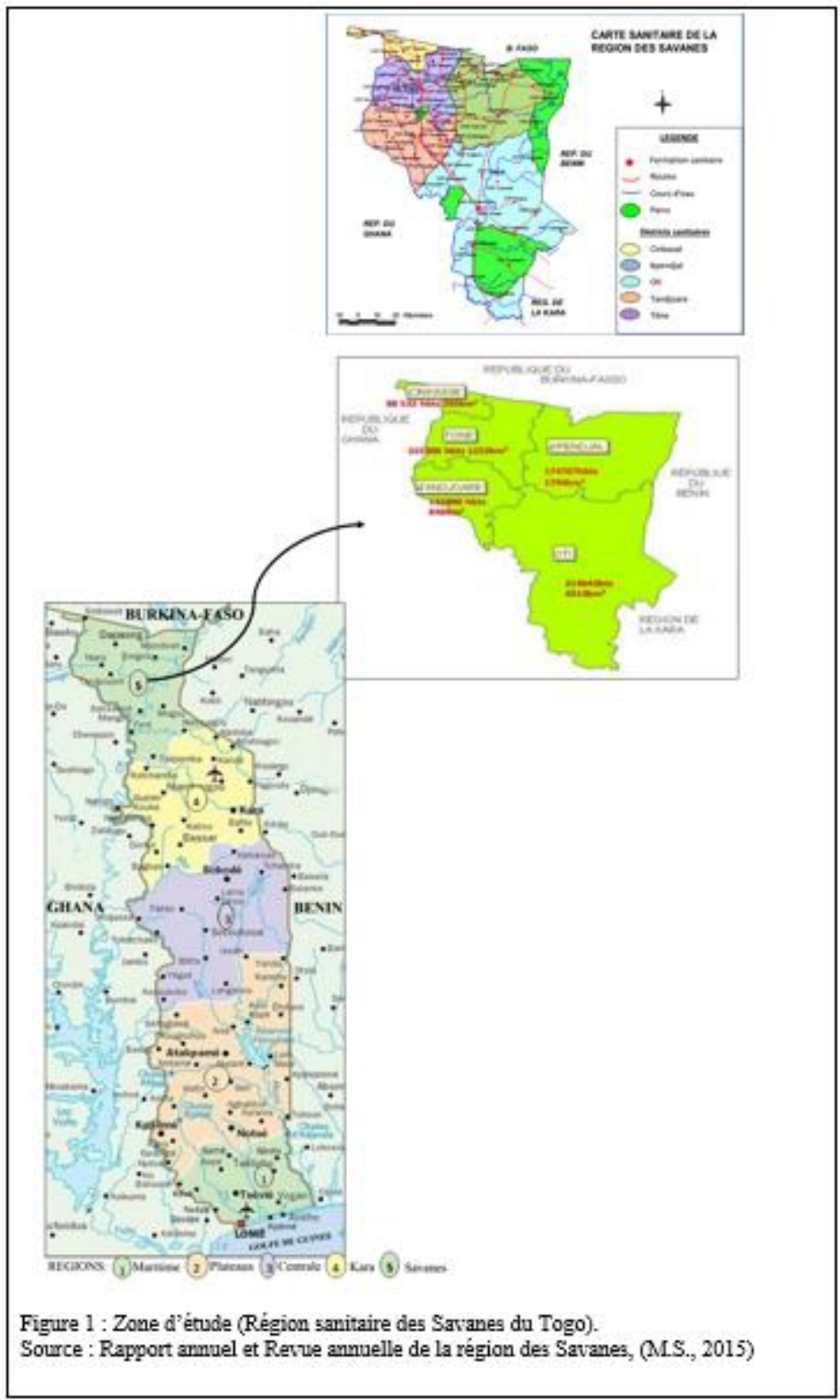

\section{Résultats}

\section{Profil sociodémographique de l'échantillon enquêté}

Cinquante un (51) praticiens de la médecine traditionnelle (43 tradithérapeutes et 8 herboristes) composés de 12 femmes et 39 hommes ont été interviewés. Leur tranche d'âge est située entre 33 ans et 110 ans avec une moyenne de 61 ans. Ils sont de niveau d'instruction diversifié, constitués de $66,67 \%$ d'analphabètes (non instruits), de 23,53\% niveau cours primaire, de $7,84 \%$ de niveau secondaire et de 1,96\% de niveau universitaire (Figure 2). 
Caractéristiques des remèdes traditionnels à base de plantes, botanique et diversités biologiques des espèces recensées

Les PMT de la Région des Savanes du Togo ont indiqué 79 recettes utilisées dans les soins de neuf pathologies liées à l'infertilité féminine. Il a été recensé 87 espèces composant ces recettes et appartenant à 48 familles botaniques dont les plus représentées sont les Combretaceae $(12,64 \%)$, les Rubiaceae $(5,7 \%)$, les Moraceae $(4,6 \%)$ et les Ceasalpiniaceae $(4,6 \%)$. Combretum ssp. (7 espèces) et Ficus ssp. (4 espèces) ont été les espèces les plus prépondérantes alors que Blighia sapida K.D.Koenig (6,48\%), Piper guineense Schumach. \& Thonn. (6,48\%), Capsicum frutescens L. (5,76\%), Zanthoxylum zanthoxyloides (Lam.) Zepern. \& Timler (5,4\%) et Aframomum melegueta K.Schum (4,32\%) ont été les espèces les plus fréquemment citées. Par ailleurs, on note une diversité de types biologiques dominée par les arbres $(36,78 \%)$, les arbustes $(27,58 \%)$ et les herbes $(24,14 \%)$ à côté desquelles les lianes $(5,75 \%)$, les épiphytes, les champignons $(2,3 \%)$ et les sous-arbrisseaux $(1,15 \%)$, plus faiblement représentés (Tableau I).

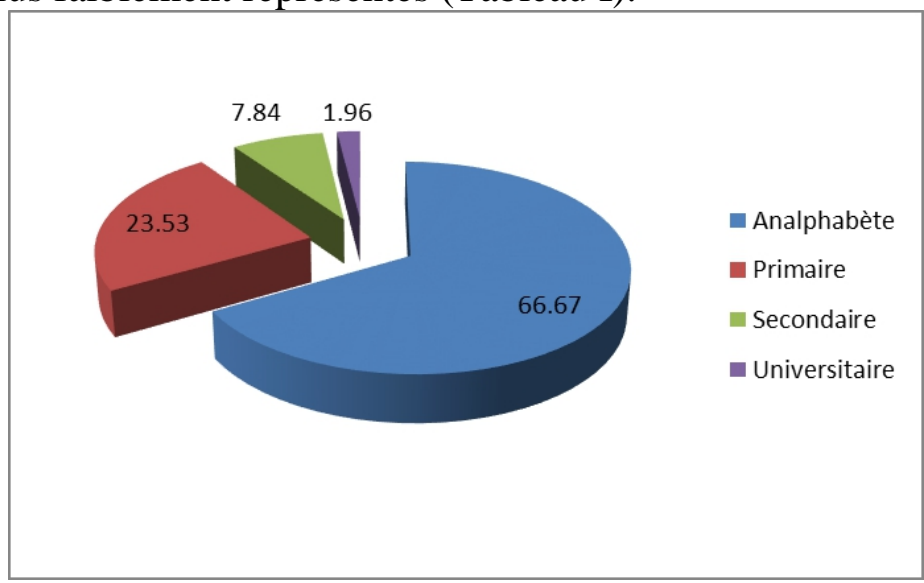

Figure 2 : Niveau d'instruction des praticiens enquêtés

\section{Données ethnopharmacologiques sur l'infertilité féminine}

\section{- Organes utilisés, modes de préparation et voies d'administration}

Les organes les plus utilisées des espèces recensées dans le traitement de l'infertilité féminine ont été essentiellement les racines $(29,17 \%)$ et les feuilles $(27,5 \%)$, (Figure 3). Les recettes dans lesquelles on retrouve ces organes sont préparées en décoction $(51,88 \%)$, en poudre $(38,12 \%)$, en macération $(5,62 \%)$, en infusion $(2,5 \%)$ et en fumigation $(1,88 \%)$ (Figure 4$)$. Elles sont administrées principalement par voie orale $(47,28 \%)$ et par bain corporel (43,48\%) (Figure 5).

- Maladies de l'infertilité et recettes de plantes utilisées pour les soigner 
Neuf pathologies liées à l'infertilité féminine parmi lesquelles les Infections sexuellement transmissibles (IST) et les Fibromes utérins sont les plus citées, ont été recensées (Tableau II). Ces pathologies ont été retrouvées dans 79 recettes dont 11 recettes à une seule espèce chacune et 68 recettes d'associations de deux à douze plantes. Ces recettes d'association de plantes comportent entre autres, 23 recettes de deux plantes, 13 recettes de trois plantes et 4 recettes de quatre plantes. Certaines de ces recettes sont présentées dans les Tableaux III et IV.

\section{- $\quad$ Facteur de consensus (FC) et Degré de fidélité (DF)}

Les valeurs du Facteur de consensus (Tableau 5) montrent que le consensus est élevé pour les IST (FC=0,61), les règles douloureuses $(\mathrm{FC}=0,56)$ et les fibromes utérins $(\mathrm{FC}=0,34)$. C'est la preuve que les praticiens maîtrisent mieux le concept de ces 3 pathologies que celui des autres. La fidélité exprimée (Tableau 6) est plus élevée pour les IST $(\mathrm{DF}=25,49 \%)$ relativement à Capsicum frutescens, Piper guineense. Elle est identique (DF=19,61\%) pour les IST et Règles douloureuses par rapport Piper guineense, Aframomum melegueta et à Zanthoxylum zanthoxyloides respectivement. Le DF est inférieur ou égal à $17,69 \%$ pour les 6 autres pathologies liées à l'infertilité féminine relativement aux 83 autres espèces recensées.

Tableau I : Diversités biologiques et ethnopharmacologiques anti-infertilité féminine

\begin{tabular}{|c|c|c|c|c|c|c|c|}
\hline Nom scientifique & Famille & $\begin{array}{c}\text { Type } \\
\text { biologiq } \\
\text { ue }\end{array}$ & $\begin{array}{c}\text { Nom en } \\
\text { langue locale }\end{array}$ & Parties utilisées & $\begin{array}{l}\text { Pathologies } \\
\text { Traitées }\end{array}$ & $\begin{array}{l}\text { Mode de } \\
\text { Préparation }\end{array}$ & $\begin{array}{l}\text { Voie } \\
\text { d'admin } \\
\text { is. }\end{array}$ \\
\hline $\begin{array}{l}\text { Abelmoschus } \\
\text { esculentus (L.) } \\
\text { Moench }\end{array}$ & $\begin{array}{c}\text { Malvace } \\
\text { ae }\end{array}$ & Herbe & Maani (moba) & Tiges & FU; IST & $\begin{array}{l}\text { Infusion; } \\
\text { Décoction }\end{array}$ & $\begin{array}{l}\text { VO; BC; } \\
\text { VV }\end{array}$ \\
\hline Adansonia digitata $\mathrm{L}$. & $\begin{array}{l}\text { Bombac } \\
\text { acae }\end{array}$ & Arbre & $\begin{array}{l}\text { Tuukala } \\
\text { (moba) }\end{array}$ & Feuilles & RD; IST & $\begin{array}{l}\text { Poudre; } \\
\text { Décoction }\end{array}$ & VO; BC \\
\hline $\begin{array}{c}\text { Aframomum melegueta } \\
\text { K.Schum }\end{array}$ & $\begin{array}{c}\text { Zingiber } \\
\text { aceae }\end{array}$ & Herbe & Jumjag (moba) & Graines & $\begin{array}{l}\text { RD; IST; TO; } \\
\text { FU }\end{array}$ & $\begin{array}{c}\text { Poudre; } \\
\text { Décoction }\end{array}$ & $\mathrm{VO} ; \mathrm{BC}$ \\
\hline $\begin{array}{c}\text { Alchornea cordifolia } \\
\text { Benth. }\end{array}$ & $\begin{array}{l}\text { Euphorb } \\
\text { iaceae }\end{array}$ & Arbuste & kpabl (moba) & Racines & $\begin{array}{l}\mathrm{RD} \\
\mathrm{IST}\end{array}$ & $\begin{array}{c}\text { Poudre; } \\
\text { Décoction }\end{array}$ & $\mathrm{VO} ; \mathrm{BC}$ \\
\hline
\end{tabular}




\begin{tabular}{|c|c|c|c|c|c|c|c|}
\hline Allium sativum $\mathrm{L}$. & $\begin{array}{c}\text { Alliacea } \\
e\end{array}$ & Herbe & Gabjag (moba) & Tiges & RD; IST; IFS & $\begin{array}{l}\text { Macération; } \\
\text { Poudre; } \\
\text { Décoction }\end{array}$ & $\begin{array}{l}\text { VO; BC; } \\
\text { VA }\end{array}$ \\
\hline Aloe vera (L.) Burm.f. & $\begin{array}{l}\text { Asphode } \\
\text { laceae }\end{array}$ & Herbe & Dôgn (moba) & Plante entière & IFS & $\begin{array}{c}\text { Poudre; } \\
\text { Décoction }\end{array}$ & VO \\
\hline $\begin{array}{c}\text { Ananas comosus (L.) } \\
\text { Merr. }\end{array}$ & $\begin{array}{c}\text { Bromeli } \\
\text { aceae }\end{array}$ & Herbe & B $\phi$ lbe (moba) & Fruits & PARF & Décoction & $\mathrm{VO}$ \\
\hline $\begin{array}{c}\text { Annona senegalensis } \\
\text { Pers. }\end{array}$ & $\begin{array}{l}\text { Annona } \\
\text { ceae }\end{array}$ & Arbuste & $\begin{array}{l}\text { Baglâwaalug/ } \\
\text { Wogl (moba) }\end{array}$ & Racines, Feuilles & $\begin{array}{l}\text { RD; IST; AR; } \\
\text { IF; IFS; FU }\end{array}$ & $\begin{array}{c}\text { Poudre; } \\
\text { Décoction }\end{array}$ & $\begin{array}{l}\text { VO; BC; } \\
\text { VV }\end{array}$ \\
\hline $\begin{array}{c}\text { Anogeissus leiocarpa } \\
\text { (DC.) Guill. \& Perr. }\end{array}$ & $\begin{array}{l}\text { Combret } \\
\text { aceae }\end{array}$ & Arbre & $\begin{array}{c}\text { Naasieg } \\
\text { (moba) }\end{array}$ & Racines, Feuilles & IST; FU & $\begin{array}{c}\text { Infusion; Poudre; } \\
\text { Décoction }\end{array}$ & $\begin{array}{l}\text { VO; BC; } \\
\text { VV }\end{array}$ \\
\hline $\begin{array}{l}\text { Balanites aegyptiaca } \\
\text { (L.) Delile }\end{array}$ & $\begin{array}{l}\text { Zygophy } \\
\text { llaceae }\end{array}$ & Arbre & $\begin{array}{c}\text { Kûkôlâkpaglig } \\
\text { (moba) }\end{array}$ & Racines & RD; IST & Décoction & $\mathrm{VO}$ \\
\hline $\begin{array}{l}\text { Blighia sapida } \\
\text { K.D.Koenig }\end{array}$ & $\begin{array}{l}\text { Sapinda } \\
\text { ceae }\end{array}$ & Arbre & Kpiig (moba) & $\begin{array}{c}\text { Ecorces de tronc, } \\
\text { Feuilles, Racines, } \\
\text { Fruits }\end{array}$ & $\begin{array}{c}\text { RD; IST; FU; } \\
\text { TO; IF; IFS; IFP }\end{array}$ & $\begin{array}{c}\text { Poudre; } \\
\text { Décoction }\end{array}$ & $\begin{array}{l}\mathrm{VO} ; \mathrm{BC} ; \\
\text { VV }\end{array}$ \\
\hline $\begin{array}{l}\text { Bombax costatum } \\
\text { Pellegr. \& Vuillet }\end{array}$ & $\begin{array}{l}\text { Bombac } \\
\text { acae }\end{array}$ & Arbre & Fuog (moba) & Feuilles & AR & Décoction & $\mathrm{VO} ; \mathrm{BC}$ \\
\hline $\begin{array}{l}\text { Calotropis procera } \\
\text { (Aiton) R.Br. }\end{array}$ & $\begin{array}{c}\text { Asclepia } \\
\text { daceae }\end{array}$ & Arbuste & $\begin{array}{c}\text { Pulâpuug } \\
\text { (moba) }\end{array}$ & Racines & RD; IST; IFS & $\begin{array}{c}\text { Poudre; } \\
\text { Décoction }\end{array}$ & $\mathrm{VO} ; \mathrm{BC}$ \\
\hline Capsicum frutescens L. & $\begin{array}{l}\text { Solanac } \\
\text { eae }\end{array}$ & $\begin{array}{c}\text { Sous- } \\
\text { arbrissea } \\
\mathrm{u} \\
\end{array}$ & Kâmi (moba) & Fruits & $\begin{array}{c}\text { IF; IST ;RD; FU; } \\
\text { TO }\end{array}$ & $\begin{array}{c}\text { Poudre; } \\
\text { Décoction }\end{array}$ & $\mathrm{VO} ; \mathrm{BC}$ \\
\hline $\begin{array}{l}\text { Carissa edulis } \\
\text { (Forssk.) Vahl }\end{array}$ & $\begin{array}{c}\text { Apocyna } \\
\text { ceae }\end{array}$ & Arbuste & $\begin{array}{l}\text { Mugulu } \\
\text { (gourma) }\end{array}$ & Racines & IST; RD & Décoction & VO \\
\hline
\end{tabular}

Note : AR : Absence de règle ; BC : Bain corporel ; FU : Fibromes utérins ; IST : Infections sexuellement transmissibles ; IF : Infertilité féminine ; IFP : Infertilité féminine primaire ; IFS : Infertilité féminine secondaire ; PARF : Purification de l'appareil reproducteur féminin ; RD : Règles douloureuses ; TO : Troubles d'ovulation; VA : Voie anale ; VO : Voie orale ; VV : Voie vaginale. 
Tableau I : Diversités biologiques et ethnopharmacologiques anti-infertilité féminine (Suite 1)

\begin{tabular}{|c|c|c|c|c|c|c|c|}
\hline Nom scientifique & Famille & $\begin{array}{c}\text { Type } \\
\text { biologiqu } \\
\mathrm{e}\end{array}$ & $\begin{array}{c}\text { Nom en } \\
\text { langue locale }\end{array}$ & $\begin{array}{l}\text { Parties } \\
\text { Utilisées }\end{array}$ & $\begin{array}{l}\text { Pathologies } \\
\text { traitées }\end{array}$ & $\begin{array}{c}\text { Mode de } \\
\text { Préparation }\end{array}$ & $\begin{array}{c}\text { Voie } \\
\text { d'admini } \\
\text { s. }\end{array}$ \\
\hline Cassia sieberiana DC. & $\begin{array}{c}\text { Caesalpini } \\
\text { aceae }\end{array}$ & Arbuste & Sâja (moba) & Racines & $\begin{array}{c}\text { IST; RD; IF; } \\
\text { AR }\end{array}$ & $\begin{array}{c}\text { Poudre; } \\
\text { Décoction }\end{array}$ & $\mathrm{VO}$ \\
\hline Cassytha filiformis L. & Lauraceae & Liane & $\begin{array}{l}\text { Dîdamokaagyi } \\
\text { cl (moba) }\end{array}$ & Plante entière & $\begin{array}{l}\text { IST; AR; } \\
\text { IFP; RD }\end{array}$ & $\begin{array}{c}\text { Poudre; } \\
\text { Décoction }\end{array}$ & $\begin{array}{c}\mathrm{VO} ; \mathrm{BC} ; \\
\mathrm{VV}\end{array}$ \\
\hline $\begin{array}{c}\text { Ceratotheca sesamoides } \\
\text { Endl. }\end{array}$ & $\begin{array}{c}\text { Pedaliacea } \\
e\end{array}$ & Herbe & Bфlun (moba) & Plante entière & FU; IF & $\begin{array}{c}\text { Macération; } \\
\text { Poudre; } \\
\text { Décoction }\end{array}$ & VO; VV \\
\hline $\begin{array}{c}\text { Chasmanthera dependens } \\
\text { Hochst. }\end{array}$ & $\begin{array}{c}\text { Menisperm } \\
\text { aceae }\end{array}$ & Liane & $\begin{array}{c}\text { Waakpel } \\
\text { (moba) }\end{array}$ & Tiges & IST; RD & $\begin{array}{c}\text { Poudre; } \\
\text { Décoction }\end{array}$ & $\begin{array}{c}\mathrm{VO} ; \mathrm{BC} ; \\
\mathrm{VV}\end{array}$ \\
\hline $\begin{array}{l}\text { Citrullus colocynthis (L.) } \\
\text { Schrad. }\end{array}$ & $\begin{array}{c}\text { Cucurbitac } \\
\text { eae }\end{array}$ & Herbe & $\begin{array}{l}\text { Nassentoutouô } \\
\mathrm{g} \text { (moba) }\end{array}$ & Fruits & $\begin{array}{l}\text { FU; IFS; } \\
\text { PARF }\end{array}$ & $\begin{array}{c}\text { Macération; } \\
\text { Poudre; } \\
\text { Décoction; } \\
\text { Fumigation }\end{array}$ & $\begin{array}{l}\mathrm{VO} ; \mathrm{BC} ; \\
\mathrm{VV}\end{array}$ \\
\hline Citrus limon (L.) Burm.f. & Rutaceae & Arbre & $\begin{array}{l}\text { Citronier } \\
\text { (français) }\end{array}$ & Fruits & $\begin{array}{l}\text { PARF; IST; } \\
\text { IFS }\end{array}$ & $\begin{array}{c}\text { Macération; } \\
\text { Décoction }\end{array}$ & VO; VA \\
\hline $\begin{array}{c}\text { Cochlospermum tinctorium } \\
\text { Perr. ex A.Rich. }\end{array}$ & $\begin{array}{c}\text { Cochlosper } \\
\text { maceae }\end{array}$ & Herbe & $\begin{array}{l}\text { Nyфgmфsaâja } \\
\text { g (moba) }\end{array}$ & Racines & $\begin{array}{l}\text { IST; FU; } \\
\text { IF }\end{array}$ & $\begin{array}{c}\text { Infusion; } \\
\text { Poudre; } \\
\text { Décoction }\end{array}$ & $\begin{array}{l}\mathrm{VO} ; \mathrm{BC} ; \\
\mathrm{VV}\end{array}$ \\
\hline $\begin{array}{c}\text { Cola nitida (Vent.) Schott } \\
\text { \& Endl. }\end{array}$ & $\begin{array}{c}\text { Sterculiace } \\
\text { ae }\end{array}$ & Arbre & $\begin{array}{r}\text { Colatier } \\
\text { (français) }\end{array}$ & Graines & $\mathrm{RD}$ & $\begin{array}{c}\text { Poudre; } \\
\text { Décoction }\end{array}$ & $\mathrm{VO} ; \mathrm{BC}$ \\
\hline $\begin{array}{c}\text { Combretum aculeatum } \\
\text { Vent. }\end{array}$ & $\begin{array}{c}\text { Combretac } \\
\text { eae }\end{array}$ & Arbuste & $\begin{array}{l}\text { Tâsuon } \\
\text { (moba) }\end{array}$ & Feuilles & IST & $\begin{array}{c}\text { Poudre; } \\
\text { Décoction }\end{array}$ & $\mathrm{VO} ; \mathrm{BC}$ \\
\hline $\begin{array}{c}\text { Combretum glutinosum } \\
\text { Perr. }\end{array}$ & $\begin{array}{c}\text { Combretac } \\
\text { eae }\end{array}$ & Arbre & $\begin{array}{c}\text { Tîtabmфn } \\
\text { (moba) }\end{array}$ & $\begin{array}{l}\text { Racines, Feuilles, } \\
\text { Ecorces de tronc }\end{array}$ & $\begin{array}{l}\text { AR; IST; FU; } \\
\text { RD; IFP }\end{array}$ & $\begin{array}{c}\text { Poudre; } \\
\text { Décoction }\end{array}$ & $\begin{array}{l}\mathrm{VO} ; \mathrm{BC} ; \\
\text { VV }\end{array}$ \\
\hline $\begin{array}{c}\text { Combretum lecardii Engl. } \\
\text { \& Diels }\end{array}$ & $\begin{array}{c}\text { Combretac } \\
\text { eae }\end{array}$ & Arbre & $\begin{array}{c}\text { Tâsuôjag } \\
\text { (moba) }\end{array}$ & Tiges & IST; RD & $\begin{array}{c}\text { Poudre; } \\
\text { Décoction }\end{array}$ & $\mathrm{VO} ; \mathrm{BC}$ \\
\hline
\end{tabular}




\begin{tabular}{|c|c|c|c|c|c|c|c|}
\hline $\begin{array}{c}\text { Combretum molle R.Br. ex } \\
\text { G.Don }\end{array}$ & $\begin{array}{c}\text { Combretac } \\
e a e\end{array}$ & Arbuste & $\begin{array}{c}\text { Jôbîbin } \\
\text { (moba) }\end{array}$ & $\begin{array}{c}\text { Racines, Feuilles, } \\
\text { Ecorces de tronc }\end{array}$ & FU; RD & $\begin{array}{c}\text { Poudre; } \\
\text { Décoction; } \\
\text { Fumigation }\end{array}$ & $\begin{array}{c}\text { VO; BC; } \\
\text { VV }\end{array}$ \\
\hline $\begin{array}{c}\text { Combretum nigricans } \\
\text { Lepr. ex Guill. \& Perr. }\end{array}$ & $\begin{array}{c}\text { Combretac } \\
\text { eae }\end{array}$ & Arbuste & $\begin{array}{c}\text { B } \phi \mathrm{ml} \\
(\mathrm{moba})\end{array}$ & $\begin{array}{c}\text { Racines, } \\
\text { Feuilles }\end{array}$ & IST; RD & $\begin{array}{c}\text { Poudre; } \\
\text { Décoction }\end{array}$ & VO; BC \\
\hline $\begin{array}{c}\text { Combretum sericeum } \\
\text { G.Don }\end{array}$ & $\begin{array}{c}\text { Combretac } \\
e a e\end{array}$ & Arbre & $\begin{array}{c}\text { Wonnaatubi } \\
\text { (moba) }\end{array}$ & $\begin{array}{c}\text { Racines, } \\
\text { Feuilles }\end{array}$ & IST; RD & $\begin{array}{c}\text { Poudre; } \\
\text { Décoction }\end{array}$ & $\begin{array}{c}\text { VO; BC; } \\
\text { VV }\end{array}$ \\
\hline Combretum ssp. & $\begin{array}{c}\text { Combretac } \\
\text { eae }\end{array}$ & Arbuste & $\begin{array}{c}\text { Tîtablâk } \phi \\
\text { (moba) }\end{array}$ & Racines & IST & $\begin{array}{c}\text { Poudre; } \\
\text { Décoction }\end{array}$ & VO; BC \\
\hline
\end{tabular}

Note : AR : Absence de règle ; $\mathrm{BC}:$ Bain corporel ; FU : Fibromes utérins ; IST : Infections sexuellement transmissibles ; IF : Infertilité féminine ; IFP : Infertilité féminine primaire ; IFS : Infertilité féminine secondaire ; PARF : Purification de l'appareil reproducteur féminin ; RD :

Règles douloureuses; TO : Troubles d'ovulation; VA : Voie anale ; VO : Voie orale ; VV : Voie vaginale.

Tableau I : Diversités biologiques et ethnopharmacologiques anti-infertilité féminine (Suite 2)

\begin{tabular}{|c|c|c|c|c|c|c|c|}
\hline Nom scientifique & Famille & $\begin{array}{c}\text { Type } \\
\text { biologiq } \\
\text { ue }\end{array}$ & $\begin{array}{c}\text { Nom en langue } \\
\text { locale }\end{array}$ & $\begin{array}{c}\text { Parties } \\
\text { Utilisées }\end{array}$ & $\begin{array}{c}\text { Pathologies } \\
\text { traitées }\end{array}$ & $\begin{array}{c}\text { Mode de } \\
\text { Préparati } \\
\text { on }\end{array}$ & $\begin{array}{c}\text { Voie } \\
\text { d'admi } \\
\text { nis. }\end{array}$ \\
\hline $\begin{array}{c}\text { Crinum seylanicum L. (Syn }: \text { C. } \\
\text { ornatum) (L.f. ex Aiton) Bury }\end{array}$ & $\begin{array}{c}\text { Amaryllid } \\
\text { aceae }\end{array}$ & Herbe & $\begin{array}{c}\text { Kankoagmougle } \\
\text { (moba) }\end{array}$ & Plante entière & $\begin{array}{c}\text { IST; FU; } \\
\text { RD; AR; } \\
\text { IFS }\end{array}$ & $\begin{array}{c}\text { Poudre; } \\
\text { Décoctio } \\
n\end{array}$ & $\begin{array}{c}\text { VO; } \\
\text { BC; } \\
\text { VV }\end{array}$ \\
\hline $\begin{array}{c}\text { Crossopteryx febrifuga (G.Don) } \\
\text { Benth. }\end{array}$ & Rubiaceae & Arbuste & Naakpabl (moba) & Racines & IFP & $\begin{array}{c}\text { Décoctio } \\
n\end{array}$ & $\begin{array}{c}\text { VO; } \\
\text { BC; } \\
\text { VV }\end{array}$ \\
\hline $\begin{array}{c}\text { Cymbopogon proximus (A.Rich.) } \\
\text { Stapf }\end{array}$ & Poaceae & Herbe & $\begin{array}{c}\text { Mocidnuuni } \\
\text { (moba) }\end{array}$ & Feuilles & AR & $\begin{array}{c}\text { Décoctio } \\
n\end{array}$ & $\begin{array}{c}\text { VO; } \\
\text { BC }\end{array}$ \\
\hline $\begin{array}{c}\text { Daniellia oliveri (Rolfe) Hutch. \& } \\
\text { Dalziel }\end{array}$ & $\begin{array}{c}\text { Caesalpin } \\
\text { iaceae }\end{array}$ & Arbuste & Nyфbg (moba) & $\begin{array}{c}\text { Racines, Feuilles, } \\
\text { Ecorces de tronc }\end{array}$ & $\begin{array}{c}\text { IST; FU; IF; } \\
\text { IFS; RD }\end{array}$ & $\begin{array}{c}\text { Poudre; } \\
\text { Décoctio } \\
n\end{array}$ & $\begin{array}{c}\text { VO; } \\
\text { BC }\end{array}$ \\
\hline
\end{tabular}




\begin{tabular}{|c|c|c|c|c|c|c|c|}
\hline $\begin{array}{c}\text { Diospyros mespiliformis Hochst. } \\
\text { ex A.DC. }\end{array}$ & $\begin{array}{c}\text { Ebenacea } \\
e\end{array}$ & Arbre & Gaabфn (moba) & Feuilles & $\begin{array}{l}\text { AR; RD; } \\
\text { IFP }\end{array}$ & $\begin{array}{c}\text { Décoctio } \\
\mathrm{n}\end{array}$ & $\begin{array}{l}\text { VO; } \\
\text { BC; } \\
\text { VV }\end{array}$ \\
\hline Entada africana Guill. \& Perr. & Fabaceae & Arbre & Pêkuag (moba) & Racines, Feuilles & $\begin{array}{l}\text { AR; TO; } \\
\text { IST; FU }\end{array}$ & $\begin{array}{c}\text { Poudre; } \\
\text { Décoctio } \\
n\end{array}$ & $\begin{array}{l}\mathrm{VO} \\
\mathrm{BC}\end{array}$ \\
\hline Euphorbia hirta L. & $\begin{array}{l}\text { Euphorbi } \\
\text { aceae }\end{array}$ & Herbe & $\begin{array}{l}\text { Lâjelgnaabiim } \\
\text { (moba) }\end{array}$ & Plante entière & IST & $\begin{array}{c}\text { Poudre; } \\
\text { Décoctio } \\
\text { n }\end{array}$ & $\begin{array}{l}\text { VO; } \\
\text { BC; } \\
\text { VV }\end{array}$ \\
\hline Feretia apodanthera Delile & Rubiaceae & Arbuste & $\begin{array}{l}\text { Naasus } \phi l g \\
\text { (moba) }\end{array}$ & $\begin{array}{l}\text { Feuilles, } \\
\text { Tiges }\end{array}$ & $\mathrm{RD} ; \mathrm{AR}$ & $\begin{array}{c}\text { Poudre; } \\
\text { Décoctio } \\
\text { n }\end{array}$ & $\begin{array}{l}\text { VO; } \\
\text { BC; } \\
\text { VV }\end{array}$ \\
\hline Ficus capensis Thunb. & Moraceae & Arbre & $\begin{array}{c}\text { Kîkâluôbeon } \\
\text { (moba) }\end{array}$ & Ecorces de tronc & $\mathrm{RD} ; \mathrm{AR}$ & $\begin{array}{c}\text { Décoctio } \\
\mathrm{n}\end{array}$ & $\mathrm{VO}$ \\
\hline Ficus ingens (Miq.) Miq. & Moraceae & Arbre & Puaфg (moba) & Feuilles & $\mathrm{RD} ; \mathrm{AR}$ & Poudre & VO \\
\hline Ficus ssp. & Moraceae & Herbe & Titutuag (moba) & Racines & AR & $\begin{array}{c}\text { Décoctio } \\
\mathrm{n}\end{array}$ & $\begin{array}{l}\mathrm{VO} ; \\
\mathrm{BC}\end{array}$ \\
\hline Ficus sycomorus L. & Moraceae & Arbre & Kîkan (moba) & Fruits & RD; IST & $\begin{array}{c}\text { Décoctio } \\
\mathrm{n}\end{array}$ & VO \\
\hline $\begin{array}{c}\text { Ganoderma lucidum (Curtis ex Fr.) } \\
\text { P.Karst. }\end{array}$ & $\begin{array}{l}\text { Ganoder } \\
\text { mataceae }\end{array}$ & $\begin{array}{c}\text { Champig } \\
\text { non }\end{array}$ & $\begin{array}{l}\text { Champignon du } \\
\text { néré (français) }\end{array}$ & $\begin{array}{l}\text { Champignon } \\
\text { Entier }\end{array}$ & IFS; IF & $\begin{array}{c}\text { Poudre; } \\
\text { Décoctio } \\
n\end{array}$ & $\begin{array}{l}\mathrm{VO} \\
\mathrm{BC}\end{array}$ \\
\hline $\begin{array}{c}\text { Ganoderma colosus }(F r .) \text { C.F. } \\
\text { Baker }\end{array}$ & $\begin{array}{l}\text { Ganoder } \\
\text { mataceae }\end{array}$ & $\begin{array}{c}\text { Champig } \\
\text { non }\end{array}$ & Madliog (moba) & $\begin{array}{l}\text { Champignon } \\
\text { Entier }\end{array}$ & $\begin{array}{l}\text { IST; FU; } \\
\text { TO; IF }\end{array}$ & $\begin{array}{c}\text { Infusion; } \\
\text { Poudre; } \\
\text { Décoctio } \\
\text { n }\end{array}$ & $\begin{array}{l}\text { VO; } \\
\text { BC; } \\
\text { VV }\end{array}$ \\
\hline $\begin{array}{c}\text { Gardenia ternifolia Schumach. \& } \\
\text { Thonn. }\end{array}$ & Rubiaceae & Arbuste & $\begin{array}{c}\text { Naasabljalâbuug } \\
\text { (moba) }\end{array}$ & Feuilles & AR & $\begin{array}{c}\text { Décoctio } \\
n\end{array}$ & $\begin{array}{l}\mathrm{VO} ; \\
\mathrm{BC}\end{array}$ \\
\hline
\end{tabular}

Note : AR : Absence de règle ; $\mathrm{BC}$ : Bain corporel ; FU : Fibromes utérins ; IST : Infections sexuellement transmissibles ; IF : Infertilité

féminine ; IFP : Infertilité féminine primaire ; IFS : Infertilité féminine secondaire ; PARF : Purification de l'appareil reproducteur féminin ; RD : Règles douloureuses; TO : Troubles d'ovulation; VA : Voie anale ; VO : Voie orale ; VV : Voie vaginale. 
Tableau I : Diversités biologiques et ethnopharmacologiques anti-infertilité féminine (Suite 3)

\begin{tabular}{|c|c|c|c|c|c|c|c|}
\hline Nom scientifique & Famille & $\begin{array}{c}\text { Type } \\
\text { biologiqu } \\
\mathrm{e}\end{array}$ & $\begin{array}{c}\text { Nom en } \\
\text { langue } \\
\text { locale }\end{array}$ & $\begin{array}{l}\text { Parties } \\
\text { Utilisées }\end{array}$ & $\begin{array}{l}\text { Pathologie } \\
\text { s traitées }\end{array}$ & $\begin{array}{c}\text { Mode de } \\
\text { Préparation }\end{array}$ & $\begin{array}{c}\text { Voie } \\
\text { d'admini } \\
\text { s. }\end{array}$ \\
\hline Indigofera pulchra Willd. & $\begin{array}{c}\text { Fabacea } \\
e\end{array}$ & Herbe & $\begin{array}{c}\text { Kpamlali } \\
\text { (moba) }\end{array}$ & Plante entière & IST; RD & Décoction & $\mathrm{VO} ; \mathrm{BC}$ \\
\hline Jatropha gossypiifolia L. & $\begin{array}{l}\text { Euphorb } \\
\text { iaceae }\end{array}$ & Arbuste & $\begin{array}{l}\text { Naâmфn } \\
\text { (moba) }\end{array}$ & Feuilles & IST & Décoction & VO \\
\hline Khaya grandifoliola C.DC. & $\begin{array}{c}\text { Meliace } \\
\text { ae }\end{array}$ & Arbre & $\begin{array}{l}\text { Kpeg-satig } \\
\text { (moba) }\end{array}$ & Racines & IST; RD & Décoction & VO \\
\hline $\begin{array}{c}\text { Khaya senegalensis (Desr.) } \\
\text { A.Juss. }\end{array}$ & $\begin{array}{c}\text { Meliace } \\
\text { ae }\end{array}$ & Arbre & $\begin{array}{l}\text { Kpeg } \\
\text { (moba) }\end{array}$ & Ecorces de tronc & AR; RD & Poudre & VO \\
\hline $\begin{array}{l}\text { Kigelia africana (Lam.) } \\
\text { Benth. }\end{array}$ & $\begin{array}{l}\text { Bignonia } \\
\text { ceae }\end{array}$ & Arbre & $\begin{array}{l}\text { Abiliou } \\
\text { (Tem) }\end{array}$ & $\begin{array}{c}\text { Ecorces de tronc } \\
\text { Racines }\end{array}$ & $\begin{array}{l}\text { IFS; IF; } \\
\text { IFP }\end{array}$ & $\begin{array}{l}\text { Macération; } \\
\text { Décoction }\end{array}$ & VO; VA \\
\hline Lannea acida A.Rich. & $\begin{array}{c}\text { Anacard } \\
\text { iaceae }\end{array}$ & Arbre & $\begin{array}{l}\text { Gbednaatun } \\
\text { (moba) }\end{array}$ & Ecorces de tronc & IST & $\begin{array}{c}\text { Poudre; } \\
\text { Décoction }\end{array}$ & $\begin{array}{l}\mathrm{VO} ; \mathrm{BC} ; \\
\text { VV }\end{array}$ \\
\hline $\begin{array}{c}\text { Lepidagathis chariensis } \\
\text { Benoist }\end{array}$ & $\begin{array}{l}\text { Acantha } \\
\text { ceae }\end{array}$ & Herbe & $\begin{array}{l}\text { Wabdûgbini } \\
\text { (moba) }\end{array}$ & Plante entière & IST; RD & Décoction & VO \\
\hline $\begin{array}{l}\text { Leptadenia hastata (Pers.) } \\
\text { Decne. }\end{array}$ & $\begin{array}{l}\text { Asclepia } \\
\text { daceae }\end{array}$ & Liane & $\begin{array}{l}\text { Naliong } \\
\text { (moba) }\end{array}$ & Racines & IST; RD & $\begin{array}{c}\text { Poudre; } \\
\text { Décoction }\end{array}$ & $\mathrm{VO} ; \mathrm{BC}$ \\
\hline Mangifera indica $\mathrm{L}$. & $\begin{array}{c}\text { Anacard } \\
\text { iaceae }\end{array}$ & Arbre & $\begin{array}{l}\text { Maфun } \\
\text { (moba) }\end{array}$ & $\begin{array}{l}\text { Feuilles, } \\
\text { Racines }\end{array}$ & AR & Décoction & $\mathrm{VO} ; \mathrm{BC}$ \\
\hline $\begin{array}{l}\text { Mitragyna inermis (Willd.) } \\
\text { Kuntze }\end{array}$ & $\begin{array}{c}\text { Rubiace } \\
\text { ae }\end{array}$ & Arbre & $\begin{array}{l}\text { Yelun } \\
\text { (moba) }\end{array}$ & Feuilles & $\begin{array}{l}\text { IST; RD; } \\
\text { FU }\end{array}$ & Décoction & $\begin{array}{l}\text { VO; BC; } \\
\text { VV }\end{array}$ \\
\hline $\begin{array}{l}\text { Monodora myristica } \\
\text { (Gaertn.) Dunal }\end{array}$ & $\begin{array}{l}\text { Annonac } \\
\text { eae }\end{array}$ & Arbre & $\begin{array}{l}\text { Azingbebia } \\
\quad(\text { Tem })\end{array}$ & Graines & $\begin{array}{c}\text { RD; IST; } \\
\text { FU; } \\
\text { TO; IF }\end{array}$ & $\begin{array}{l}\text { Poudre; } \\
\text { Décoction }\end{array}$ & $\mathrm{VO} ; \mathrm{BC}$ \\
\hline Musa ssp. & $\begin{array}{c}\text { Musacea } \\
e\end{array}$ & Herbe & $\begin{array}{l}\text { Kuadu } \\
\text { (moba) }\end{array}$ & Fruits & $\mathrm{RD}$ & $\begin{array}{c}\text { Poudre; } \\
\text { Décoction }\end{array}$ & VO \\
\hline Ocimum canum Sims & $\begin{array}{c}\text { Lamiace } \\
\text { ae }\end{array}$ & Herbe & $\begin{array}{c}\text { Fuomane } \\
\text { (moba) }\end{array}$ & Feuilles & AR; RD & Poudre & VO \\
\hline
\end{tabular}




\begin{tabular}{|c|c|c|c|c|c|c|c|}
\hline $\begin{array}{c}\text { Opilia celtidifolia (Guill. \& } \\
\text { Perr.) Endl. ex Walp. }\end{array}$ & $\begin{array}{c}\text { Opiliace } \\
a e\end{array}$ & Liane & $\begin{array}{c}\text { Wâmpmiim } \\
\text { (moba) }\end{array}$ & Plante entière & IST; FU & $\begin{array}{c}\text { Poudre; } \\
\text { Décoction }\end{array}$ & VO; BC \\
\hline $\begin{array}{c}\text { Parkia biglobosa (Jacq.) } \\
\text { R.Br. ex G.Don }\end{array}$ & $\begin{array}{c}\text { Mimosac } \\
e a e\end{array}$ & Arbre & $\begin{array}{c}\text { Duug } \\
\text { (moba) }\end{array}$ & $\begin{array}{c}\text { Fruits, Tiges, Ecorces } \\
\text { de tronc, Feuilles }\end{array}$ & $\begin{array}{c}\text { RD; FU; } \\
\text { IST; } \\
\text { IF }\end{array}$ & $\begin{array}{c}\text { Poudre; } \\
\text { Décoction; } \\
\text { Fumigation }\end{array}$ & $\begin{array}{c}\text { VO; BC; } \\
\text { VV }\end{array}$ \\
\hline
\end{tabular}

Note : AR : Absence de règle ; $\mathrm{BC}$ : Bain corporel ; FU : Fibromes utérins ; IST : Infections sexuellement transmissibles ; IF : Infertilité féminine ; IFP : Infertilité féminine primaire ; IFS : Infertilité féminine secondaire ; PARF : Purification de l'appareil reproducteur féminin ; RD : Règles douloureuses ; TO : Troubles d'ovulation; VA : Voie anale ; VO : Voie orale ; VV : Voie vaginale.

Tableau I : Diversités biologiques et ethnopharmacologiques anti-infertilité féminine (Suite 4)

\begin{tabular}{|c|c|c|c|c|c|c|c|}
\hline Nom scientifique & Famille & $\begin{array}{c}\text { Type } \\
\text { biologiqu } \\
\mathrm{e}\end{array}$ & $\begin{array}{c}\text { Nom en } \\
\text { langue locale }\end{array}$ & $\begin{array}{l}\text { Parties } \\
\text { Utilisées }\end{array}$ & $\begin{array}{l}\text { Pathologies } \\
\text { traitées }\end{array}$ & $\begin{array}{c}\text { Mode de } \\
\text { Préparatio } \\
\text { n }\end{array}$ & $\begin{array}{c}\text { Voie } \\
\text { d'admini } \\
\text { s. }\end{array}$ \\
\hline Paullinia pinnata $\mathrm{L}$. & $\begin{array}{c}\text { Sapindace } \\
\text { ae }\end{array}$ & Arbuste & $\begin{array}{c}\text { Sabug } \\
\text { (moba) }\end{array}$ & $\begin{array}{l}\text { Feuilles, Ecorces de } \\
\text { tronc, Racines }\end{array}$ & $\begin{array}{l}\text { IF; IST; RD; } \\
\text { IFS; IFP }\end{array}$ & $\begin{array}{c}\text { Poudre; } \\
\text { Décoction }\end{array}$ & $\mathrm{VO} ; \mathrm{BC}$ \\
\hline $\begin{array}{c}\text { Piliostigma thonningii } \\
\text { (Schumach.) Milne-Redh. }\end{array}$ & $\begin{array}{c}\text { Caesalpin } \\
\text { iaceae }\end{array}$ & Arbuste & $\begin{array}{c}\text { Naabagin } \\
\text { (moba) }\end{array}$ & Racines, Feuilles & IF; AR & $\begin{array}{l}\text { Poudre; } \\
\text { Décoction }\end{array}$ & $\mathrm{VO} ; \mathrm{BC}$ \\
\hline $\begin{array}{c}\text { Piper guineense Schumach. \& } \\
\text { Thonn. }\end{array}$ & $\begin{array}{c}\text { Piperacea } \\
e\end{array}$ & Liane & $\begin{array}{c}\text { Mousoulou } \\
(\mathrm{moba})\end{array}$ & Fruits & $\begin{array}{l}\text { IST; RD; FU; } \\
\text { TO; IF; AR }\end{array}$ & $\begin{array}{l}\text { Poudre; } \\
\text { Décoction }\end{array}$ & $\mathrm{VO} ; \mathrm{BC}$ \\
\hline Pterocarpus erinaceus Poir. & $\begin{array}{l}\text { Papiliona } \\
\text { ceae }\end{array}$ & Arbre & $\begin{array}{l}\text { Naaton } \\
\text { (moba) }\end{array}$ & Ecorces de tronc & IST & Décoction & $\mathrm{VO}$ \\
\hline $\begin{array}{c}\text { Sarcocephalus latifolius (Sm.) } \\
\text { E.A.Bruce }\end{array}$ & Rubiaceae & Arbuste & $\begin{array}{c}\text { Guon } \\
(\text { moba })\end{array}$ & $\begin{array}{l}\text { Fruits, Racines, } \\
\text { Feuilles } \\
\end{array}$ & $\begin{array}{l}\text { IST; RD; } \\
\text { FU; IF }\end{array}$ & $\begin{array}{l}\text { Poudre; } \\
\text { Décoction }\end{array}$ & $\mathrm{VO}$ \\
\hline $\begin{array}{c}\text { Sclerocarya birrea (A.Rich.) } \\
\text { Hochst. }\end{array}$ & $\begin{array}{c}\text { Anacardia } \\
\text { ceae }\end{array}$ & Arbre & $\begin{array}{c}\text { Naanyicn } \\
\text { (moba) }\end{array}$ & Racines, Feuilles & TO & $\begin{array}{l}\text { Poudre; } \\
\text { Décoction }\end{array}$ & $\mathrm{VO} ; \mathrm{BC}$ \\
\hline $\begin{array}{l}\text { Securidaca longipedunculata } \\
\text { Fresen. }\end{array}$ & $\begin{array}{l}\text { Polygalac } \\
\text { eae }\end{array}$ & Arbre & Palg (moba) & Racines & $\begin{array}{l}\text { RD; AR; IST; } \\
\text { FU; PARF }\end{array}$ & $\begin{array}{c}\text { Macératio } \\
\text { n; } \\
\text { Poudre; } \\
\text { Décoction }\end{array}$ & VO \\
\hline Sorghum bicolor $($ L.) Moench & Poaceae & Herbe & Cali (moba) & Graines & $\begin{array}{l}\text { IST; RD; } \\
\text { IFP; IF }\end{array}$ & $\begin{array}{l}\text { Poudre; } \\
\text { Décoction }\end{array}$ & $\mathrm{VO} ; \mathrm{BC}$ \\
\hline
\end{tabular}




\begin{tabular}{|c|c|c|c|c|c|c|c|}
\hline $\begin{array}{c}\text { Stereospermum kunthianum } \\
\text { Cham. }\end{array}$ & $\begin{array}{l}\text { Bignoniac } \\
\text { eae }\end{array}$ & Arbuste & $\begin{array}{l}\text { Naalîliig } \\
\text { (moba) }\end{array}$ & $\begin{array}{l}\text { Feuilles, Ecorces de } \\
\text { tronc }\end{array}$ & FU & Poudre & $\mathrm{VO}$ \\
\hline $\begin{array}{c}\text { Striga gesnerioides (Willd.) } \\
\text { Vatke }\end{array}$ & $\begin{array}{l}\text { Scrophula } \\
\text { riaceae }\end{array}$ & Herbe & Yam (moba) & Fruits & $\mathrm{FU}$ & $\begin{array}{c}\text { Macératio } \\
\text { n; } \\
\text { Décoction }\end{array}$ & VO; VV \\
\hline Strychnos spinosa Lam. & $\begin{array}{c}\text { Loganiace } \\
\text { ae }\end{array}$ & Arbre & $\begin{array}{l}\text { Pûpuasimad } \\
\text { g (moba) }\end{array}$ & Racines & IST & Poudre & $\mathrm{VO}$ \\
\hline $\begin{array}{c}\text { Syzygium aromaticum (L.) } \\
\text { Merr. \& L.M.Perry }\end{array}$ & Myrtaceae & Arbre & $\begin{array}{c}\text { Kanafru } \\
\text { (kotokoli) }\end{array}$ & Fruits & $\begin{array}{l}\text { IST; AR; } \\
\text { RD; IF }\end{array}$ & Décoction & $\mathrm{VO}$ \\
\hline $\begin{array}{c}\text { Tacca involucrata Schumach. } \\
\text { \& Thonn. }\end{array}$ & Taccaceae & Herbe & $\begin{array}{c}\text { Nâpaa } \phi g \\
\text { (moba) }\end{array}$ & Tiges & IF & Poudre & $\mathrm{VO}$ \\
\hline Tamarindus indica L. & $\begin{array}{c}\text { Caesalpin } \\
\text { iaceae }\end{array}$ & Arbre & Puug (moba) & Fruits, Feuilles & $\begin{array}{l}\text { IST; AR; } \\
\text { RD }\end{array}$ & Décoction & $\mathrm{VO} ; \mathrm{BC}$ \\
\hline $\begin{array}{c}\text { Tapinanthus bangwensis (Engl. } \\
\text { \& K.Krause) Danser }\end{array}$ & $\begin{array}{l}\text { Loranthac } \\
\text { eae }\end{array}$ & Epiphyte & $\begin{array}{l}\text { Wûwan } \\
\text { (moba) }\end{array}$ & Plante entière & AR; IFS & $\begin{array}{c}\text { Poudre; } \\
\text { Décoction }\end{array}$ & $\begin{array}{l}\mathrm{VO} ; \mathrm{BC} ; \\
\text { VV }\end{array}$ \\
\hline
\end{tabular}

Note : AR : Absence de règle ; $\mathrm{BC}:$ Bain corporel ; FU : Fibromes utérins ; IST : Infections sexuellement transmissibles ; IF : Infertilité féminine ; IFP : Infertilité féminine primaire ; IFS : Infertilité féminine secondaire ; PARF : Purification de l'appareil reproducteur féminin ; RD : Règles douloureuses; TO : Troubles d'ovulation; VA : Voie anale ; VO : Voie orale ; VV : Voie vaginale.

Tableau I : Diversités biologiques et ethnopharmacologiques anti-infertilité féminine (Suite 5 et fin)

\begin{tabular}{|c|c|c|c|c|c|c|c|}
\hline Nom scientifique & Famille & $\begin{array}{c}\text { Type } \\
\text { biologiqu } \\
\mathrm{e}\end{array}$ & $\begin{array}{c}\text { Nom en } \\
\text { langue locale }\end{array}$ & $\begin{array}{c}\text { Parties } \\
\text { utilisées }\end{array}$ & $\begin{array}{l}\text { Pathologies } \\
\text { Traitées }\end{array}$ & $\begin{array}{l}\text { Mode de } \\
\text { Préparation }\end{array}$ & $\begin{array}{c}\text { Voie } \\
\text { d'adminis }\end{array}$ \\
\hline Tapinanthus ssp. & $\begin{array}{l}\text { Loranth } \\
\text { aceae }\end{array}$ & Epiphyte & $\begin{array}{l}\text { Wûwan } \\
\text { (moba) }\end{array}$ & $\begin{array}{l}\text { Plante } \\
\text { entière }\end{array}$ & IFP & $\begin{array}{l}\text { Poudre; } \\
\text { Décoction }\end{array}$ & VO \\
\hline Terminalia albida Scott-Elliot & $\begin{array}{l}\text { Combret } \\
\text { aceae }\end{array}$ & Arbuste & Kouii (bissa) & Racines & $\mathrm{FU}$ & Décoction & $\begin{array}{l}\text { VO; BC; } \\
\text { VV }\end{array}$ \\
\hline $\begin{array}{c}\text { Terminalia avicennioides Guill. \& } \\
\text { Perr. }\end{array}$ & $\begin{array}{l}\text { Combret } \\
\text { aceae }\end{array}$ & Arbuste & $\begin{array}{l}\text { Tîtabpien } \\
\text { (moba) }\end{array}$ & $\begin{array}{l}\text { Racines, } \\
\text { Feuilles }\end{array}$ & $\begin{array}{l}\text { IST; RD; } \\
\text { IF; FU }\end{array}$ & $\begin{array}{l}\text { Poudre; } \\
\text { Décoction }\end{array}$ & $\begin{array}{l}\mathrm{VO} ; \mathrm{BC} ; \\
\text { VV }\end{array}$ \\
\hline
\end{tabular}




\begin{tabular}{|c|c|c|c|c|c|c|c|}
\hline $\begin{array}{l}\text { Terminalia macroptera Guill. \& } \\
\text { Perr. }\end{array}$ & $\begin{array}{l}\text { Combret } \\
\text { aceae }\end{array}$ & Arbre & 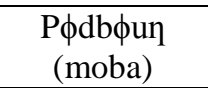 & $\begin{array}{l}\text { Racines, } \\
\text { Feuilles }\end{array}$ & IF & $\begin{array}{c}\text { Poudre; } \\
\text { Décoction }\end{array}$ & $\mathrm{VO} ; \mathrm{BC}$ \\
\hline Trichilia emetica Vahl & $\begin{array}{c}\text { Meliace } \\
\text { ae }\end{array}$ & Arbre & 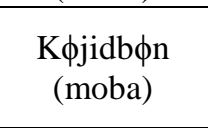 & $\begin{array}{l}\text { Ecorces } \\
\text { de tronc }\end{array}$ & IFP; IFS & $\begin{array}{c}\text { Macération } \\
; \\
\text { Décoction }\end{array}$ & VO; VA \\
\hline Vitex doniana Sweet & $\begin{array}{l}\text { Verbena } \\
\text { ceae }\end{array}$ & Arbre & Naan (moba) & $\begin{array}{l}\text { Racines, } \\
\text { Feuilles }\end{array}$ & TO & $\begin{array}{c}\text { Poudre; } \\
\text { Décoction }\end{array}$ & $\mathrm{VO} ; \mathrm{BC}$ \\
\hline Ximenia americana $\mathrm{L}$. & $\begin{array}{l}\text { Olacace } \\
\text { ae }\end{array}$ & Arbuste & $\begin{array}{l}\text { Wôgaag } \\
\text { (moba) }\end{array}$ & $\begin{array}{l}\text { Racines, } \\
\text { Feuilles }\end{array}$ & $\begin{array}{l}\text { IF; IST; } \\
\text { RD }\end{array}$ & $\begin{array}{l}\text { Poudre; } \\
\text { Décoction }\end{array}$ & $\mathrm{VO} ; \mathrm{BC}$ \\
\hline $\begin{array}{c}\text { Xylopia aethiopica (Dunal) } \\
\text { A.Rich. }\end{array}$ & $\begin{array}{c}\text { Annonac } \\
\text { eae }\end{array}$ & Arbre & $\begin{array}{l}\text { Lâjobфnu } \\
\text { (moba) }\end{array}$ & Fruits & $\begin{array}{l}\text { IST; RD; } \\
\text { FU; IF; AR }\end{array}$ & $\begin{array}{c}\text { Poudre; } \\
\text { Décoction }\end{array}$ & $\mathrm{VO} ; \mathrm{BC}$ \\
\hline $\begin{array}{l}\text { Zanthoxylum zanthoxyloides } \\
\text { (Lam.) Zepern. \& Timler }\end{array}$ & $\begin{array}{c}\text { Rutacea } \\
e\end{array}$ & Arbuste & Polg (moba) & Racines & $\begin{array}{l}\text { IST; RD; FU; TO; IF; } \\
\text { AR; IFP; IFS }\end{array}$ & $\begin{array}{c}\text { Macération } \\
; \\
\text { Poudre; } \\
\text { Décoction }\end{array}$ & $\begin{array}{l}\text { VO; BC; } \\
\text { VA }\end{array}$ \\
\hline Zea mays $\mathrm{L}$. & Poaceae & Herbe & $\begin{array}{l}\text { Kpabda } \\
\text { (moba) }\end{array}$ & Graines & IST; FU & $\begin{array}{c}\text { Poudre; } \\
\text { Décoction }\end{array}$ & VO; BC \\
\hline Zingiber officinale Roscoe & $\begin{array}{l}\text { Zingiber } \\
\text { aceae }\end{array}$ & Herbe & $\begin{array}{l}\text { Kakaadulu } \\
\text { (moba) }\end{array}$ & Tiges & $\begin{array}{l}\text { PARF; IST; } \\
\text { FU; TO; IF }\end{array}$ & $\begin{array}{l}\text { Poudre; } \\
\text { Décoction }\end{array}$ & $\mathrm{VO} ; \mathrm{BC}$ \\
\hline Zizyphus mauritiana Lam. & $\begin{array}{l}\text { Rhamna } \\
\text { ceae }\end{array}$ & Arbuste & $\begin{array}{l}\text { Kûkôkâlфgba } \\
\text { d (moba) }\end{array}$ & $\begin{array}{l}\text { Racines, } \\
\text { Feuilles }\end{array}$ & IST; RD; IFP & $\begin{array}{l}\text { Poudre; } \\
\text { Décoction }\end{array}$ & $\mathrm{VO} ; \mathrm{BC}$ \\
\hline
\end{tabular}

Note : AR : Absence de règle ; $\mathrm{BC}$ : Bain corporel ; FU : Fibromes utérins ; IST : Infections sexuellement transmissibles ; IF : Infertilité féminine ; IFP : Infertilité féminine primaire; IFS : Infertilité féminine secondaire ; PARF : Purification de l'appareil reproducteur féminin ; RD : Règles douloureuses ; TO : Troubles d'ovulation; VA : Voie anale ; VO : Voie orale ; VV : Voie vaginale. 


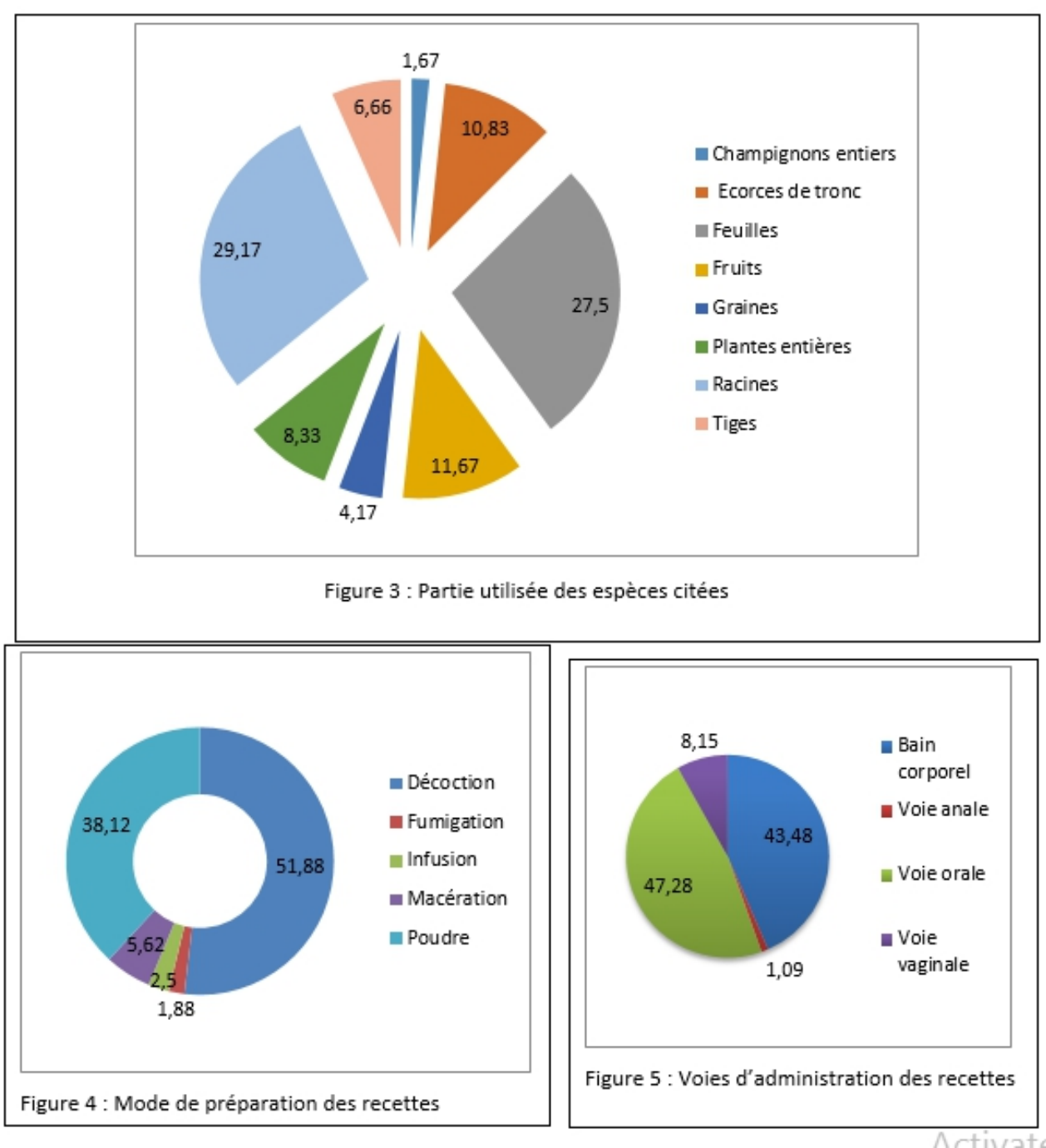


Tableau II : Pathologies d'infertilité soignées dans la Région des Savanes

\begin{tabular}{|c|c|}
\hline Pathologies & Nombre de citations \\
\hline Infections Sexuellement Transmissibles (IST) & 143 \\
\hline Règles douloureuses liées aux infertilités féminines & 408 \\
\hline Fibromes utérins & 29 \\
\hline Infertilité féminine (toute cause) & 28 \\
\hline Absence de règles (Aménorrhée) & 17 \\
\hline Infertilité féminine secondaire (toute cause) & 13 \\
\hline Infertilité féminine primaire (toute cause) & 11 \\
\hline Trouble d'ovulation & 5 \\
\hline Purification de l'appareil reproducteur féminin & 487 \\
\hline
\end{tabular}

Tableau III : Quelques recettes de plantes uniques

\begin{tabular}{|c|c|c|c|}
\hline Espèces & Parties utilisées & Modes de préparation & Voies d'administration et Posologie \\
\hline Ganoderma colossus & Champignon entier & Infusion & $\begin{array}{c}\text { Orale et } \\
1 \mathrm{VB} / \mathrm{j} \text { pendant } 3 \mathrm{~s}\end{array}$ \\
\hline Parkia biglobosa & Ecorce de tronc & Poudre & $\begin{array}{c}\text { Orale et } \\
1 \mathrm{cs} / \mathrm{j} \text { pendant } 30 \mathrm{j}\end{array}$ \\
\hline Blighia sapida & Feuilles & Décoction & Orale $1 \mathrm{VB} 3 \mathrm{x} / \mathrm{j}$ \\
\hline Cochlospermum tinctorium & Racines & Infusion & $\begin{array}{c}\text { Orale et } \\
2 \mathrm{x} / \mathrm{j} \text { pendant } 7 \mathrm{j}\end{array}$ \\
\hline Kigelia africana & Racines & Macération & $\begin{array}{l}\text { Orale et } \\
1 \mathrm{ccl} 2 \mathrm{x} / \mathrm{j}\end{array}$ \\
\hline Annona senegalensis & Feuilles et Racines & Décoction & $\begin{array}{c}\text { Orale et } \\
1 \mathrm{VB} / \mathrm{j} \text { lors des menstruations }\end{array}$ \\
\hline
\end{tabular}




\begin{tabular}{|c|c|c|c|}
\hline Mangifera indica & Feuilles & Décoction & $\begin{array}{c}\text { Orale/ BC et } \\
2 \mathrm{x} / \mathrm{j} \text { pendant } 7 \mathrm{j}\end{array}$ \\
\hline Combretum glutinosum & Feuilles et Racines & Décoction & $\begin{array}{l}\text { Orale/ BC et } \\
2 \mathrm{x} / \mathrm{j} \text { pendant } 7 \mathrm{j}\end{array}$ \\
\hline Ceratotheca sesamoides & Plante entière & Poudre & $\begin{array}{c}\text { Orale et } \\
1 \mathrm{cc} \text { ou } 1 \mathrm{cs} \text { pendant une semaine }\end{array}$ \\
\hline Daniellia oliveri & Racines & Décoction & Orale et $1 \mathrm{VB} 2 \mathrm{x} / \mathrm{j}$ pendant $7 \mathrm{j}$ \\
\hline
\end{tabular}

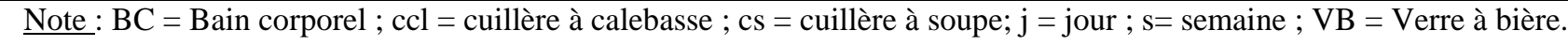

Tableau IV : Quelques recettes d'associations de plantes

\begin{tabular}{|c|c|c|c|}
\hline Espèces & Parties utilisées & Modes de préparation & $\begin{array}{c}\text { Voies d'administration et } \\
\text { Posologie }\end{array}$ \\
\hline Pterocarpus erinaceus & Ecorce & \multirow{3}{*}{ Décoction } & \multirow{3}{*}{$\begin{array}{l}\text { Orale et } \\
1 \mathrm{VM} 2 \mathrm{x} / \mathrm{j}\end{array}$} \\
\hline Syzygium aromaticum & Fruits & & \\
\hline Piper guineense & Baies & & \\
\hline Mitragyna inermis & Feuilles & \multirow{2}{*}{ Décoction } & \multirow{2}{*}{$\begin{array}{l}\text { Orale et } \\
\text { BAV }\end{array}$} \\
\hline Blighia sapida & Feuilles & & \\
\hline Annona senegalensis & Racines & \multirow{3}{*}{ Décoction } & \multirow{3}{*}{$\begin{array}{c}\text { Orale et } \\
1 \mathrm{VB} / \mathrm{j} \text { pendant } 3 \mathrm{~s}\end{array}$} \\
\hline Securidaca longipedunculata & Racines & & \\
\hline Ficus capensis & Ecorce & & \\
\hline Entada africana & Racines & \multirow{2}{*}{ Décoction } & \multirow{2}{*}{$\begin{array}{c}\text { Orale et } \\
1 \mathrm{VB} \text { en prise unique }\end{array}$} \\
\hline Combretum glutinosum & Racines & & \\
\hline Annona senegalensis & Feuilles et Racines & \multirow{2}{*}{ Décoction } & - BC et $2 x / j$ \\
\hline Danniellia oliveri & Feuilles et Racines & & - Orale et $1 \mathrm{VB} 2 \mathrm{x} / \mathrm{j}$ (pendant $7 \mathrm{j}$ ) \\
\hline Sarcocephalus latifolius & Fruits & Décoction & Orale et \\
\hline
\end{tabular}




\begin{tabular}{|c|c|c|c|}
\hline Ficus sycomorus & Fruits & & $1 \mathrm{VB} 3 \mathrm{x} / \mathrm{j}$ \\
\hline Blighia sapida & Feuilles & \multirow{3}{*}{ Décoction } & \multirow{5}{*}{$\begin{array}{c}- \text { BC: } 2 \mathrm{x} / \mathrm{j} \text { pendant } 7 \mathrm{j} \\
\text { Orale et } \\
1 \mathrm{cs} 2 \mathrm{x} / \mathrm{j} \text { en période de menstruation }\end{array}$} \\
\hline Terminalia avicennoides & Feuilles & & \\
\hline Colocynthis citrullus & Fruits & & \\
\hline Ziziphus mauritiana & Racines & \multirow{2}{*}{ Décoction } & \\
\hline Sorghum bicolor & Graines & & \\
\hline Stereospermum kunthianum & Feuilles & \multirow{2}{*}{ Poudre } & \multirow{2}{*}{$\begin{array}{c}\text { Orale et } \\
2 \mathrm{cc} 2 \mathrm{x} / \mathrm{j} \text { pendant } 3 \mathrm{j}\end{array}$} \\
\hline Securidaca longipedunculata & Racines & & \\
\hline Daniellia oliveri & Ecorce & \multirow{2}{*}{ Décoction } & \multirow{2}{*}{$\begin{array}{l}\text { Orale et } \\
1 \mathrm{VM} 2 \mathrm{x} / \mathrm{j}\end{array}$} \\
\hline Zanthoxylum zanthoxyloides & Ecorce & & \\
\hline
\end{tabular}

Note $: \mathrm{BAV}=$ Boire à volonté $; \mathrm{BC}=$ Bain corporel $; \mathrm{cs}=$ cuillère à soupe $; \mathrm{j}=$ jour ;

$\mathrm{s}=$ semaine $; \mathrm{VB}=$ Verre à bière $; \mathrm{VM}=$ Verre à madère.

Tableau V : Facteur de consensus des pathologies liées à l'infertilité féminine

\begin{tabular}{|c|c|}
\hline Pathologies & FC \\
\hline Infections Sexuellement Transmissibles (IST) & 0,61 \\
\hline Règles douloureuses & 0,56 \\
\hline Fibromes utérins & 0,34 \\
\hline Infertilité féminine & 0,18 \\
\hline Absence de règles & 0,15 \\
\hline Infertilité féminine secondaire & 0,125 \\
\hline Infertilité féminine primaire & 0,08 \\
\hline Purification de l'appareil reproducteur féminin & 0 \\
\hline Trouble d'ovulation & 0 \\
\hline
\end{tabular}


Tableau VI : Degré de fidélité des plantes à quelques pathologies

\begin{tabular}{|c|c|c|c|}
\hline Espèces & Pathologies & $\begin{array}{c}\text { Nombre de } \\
\text { praticiens }\end{array}$ & DF (\%) \\
\hline Capsicum frutescens & IST & 13 & 25,49 \\
\hline Piper guineense & IST & 13 & 25,49 \\
\hline Aframomum melegueta & IST & 10 & 19,61 \\
\hline Piper guineense & $\begin{array}{c}\text { Règles } \\
\text { douloureuses }\end{array}$ & 10 & 19,61 \\
\hline $\begin{array}{c}\text { Zanthoxylum } \\
\text { zanthoxyloides }\end{array}$ & IST & 10 & 19,61 \\
\hline
\end{tabular}

\section{Plantes sélectionnées pour tests de laboratoire}

Trois espèces exclusives parmi les recettes de plantes uniques (Ganoderma colossus, Kigelia africana et Mangifera indica) ont été sélectionnées par la SEP. Par la SEPA et le DF, Blighia sapida, bien que plante unique mais se retrouvant dans des recettes d'association de 2 à 4 plantes antiinfertilités, a été retenue. Ces quatre plantes feront l'objet de futurs travaux sur des modèles de laboratoire pour évaluer leurs propriétés anti-infertilité féminine.

\section{Discussion}

Il s'agit d'une première étude du genre réalisée dans cette zone d'étude du Togo en ce qui concerne l'infertilité féminine.

L'enquête ethnobotanique a été réalisée auprès de 51 praticiens de la région, qui étaient majoritairement des séniors de sexe masculin. Ce profil des praticiens de la région sanitaire des Savanes du Togo est celui observé dans la plupart des études similaires, confirmant que la pratique de la médecine traditionnelle est l'apanage des hommes d'âge mûr (Adjanohoun et al., 1986, 1989 ; Koudouvo, 2009, MacDonald, et al., 2016). Ce travail qui est une étude préliminaire, a contribué, à partir d'enquêtes ethnobotaniques réalisées chez les tradithérapeutes et herboristes, à l'obtention d'une base de données sur les espèces anti-infertilité du Togo.

Les données recueillies ont montré une importante diversité des plantes et recettes de plantes utilisées dans le traitement de l'infertilité féminine dans la zone d'étude : 87 espèces végétales appartenant à 48 familles et 79 recettes ont été recensées. Des études ethnobotaniques précédentes sur les plantes antiinfertilté ont recensées 75 plantes appartenant à 41 familles (Soladoye et al., 2014), 33 espèces provenant de 19 familles (Telefo, et al., 2011) et 25 plantes regroupées en 14 familles (Hadj-Seyd et al., 2015), ce qui confirme nos résultats.

En Afrique en général et particulièrement dans les sous-régions de l'Afrique Occidentale et Centrale, une riche diversité biologique médicinale 
anti-infertilité féminine a été répertoriée. Ainsi au Nigéria, 15 espèces sur les 87 recensées par la présente étude, avaient déjà été citées dans des études similaires (Soladoye et al., 2014). Les feuilles de Piper guineense y sont utilisées pour corriger des problèmes d'infertilité féminine alors que Zingiber officinale et d'Aframomum melegueta collectées par nos travaux, ont été utilisées comme aphrodisiaques au Cameroun (Noumi, et al., 1998, Iwu, 1993). L'usage d'Annona senegalensis comme plante anti-infertilité a été précédemment rapporté (Batawila et al., 2007, Deleke, 2005, Göhre, et al., 2016, Ouattara, et al., 2016). Kigelia africana a été signalées comme antiinfertilité (Batawila et al., 2007, Deleke, 2005). Daniellia oliveri, Pterocarpus erinaceus, Crossopteryx febrifuga ont été citées pour leurs propriétés antiinfertilité (Deleke, 2005). Mangifera indica et Khaya grandifoliola ont également été citées pout leur utilisation en médecine traditionnelle pour traiter l'infertilité (Soladoye et al., 2014). De ces travaux, seules Annona senegalensis et Kigelia africana (Batawila et al., 2007) avaient été citées par des enquêtes ethnobotaniques réalisées au Togo. Toutes les autres espèces cidessus ayant été recensées par nos travaux, il en ressort une similitude dans les résultats et la preuve de l'existence au Togo de connaissances endogènes sur le traitement de l'infertilité féminine.

La fréquence de certaines espèces et la convergence de vue des praticiens pour leurs utilisations dans le traitement de certaines pathologies anti-infertilité féminine sont assez significatives. Ainsi les fréquences d'utilisation de chaque plante montrent que les espèces les plus utilisées par les praticiens de la Région sanitaire des Savanes pour soigner l'infertilité féminine sont Blighia sapida, Piper guineense, Capsicum frutescens, Zanthoxylum zanthoxyloides et Aframomum melegueta. Ces résultats traduiraient tout au moins l'importance de ces cinq espèces dans le traitement de l'infertilité chez la femme.

Les parties végétales les plus utilisées des plantes par les praticiens dans cette étude ont été la racine à 29,17\%. Ajouté aux taux de 1'écorce de tronc $(10,83 \%)$ et de la plante entière $(8,33 \%)$, les organes vitaux non renouvelables représentent 48,33\% des partie utilisées des espèces recensées, ce qui constitue une véritable menace de la biodiversité médicinale. Des études récentes au Togo (Koudouvo et $a l ., 2017$ ) ont révélées la menace des diversités biologiques médicinales par la pression anthropique relativement au prélèvement de ces organes. L'éducation aux bonnes pratiques d'utilisation des parties renouvelables (feuilles et fruits) à la place des racines et de l'écorce de tronc, contribuera à la préservation de la biodiversité. L'utilisation des feuilles à $27,5 \%$ peut être expliquée par leur abondance, l'aisance et la rapidité de la récolte.

La valeur des Facteurs de consensus ont montré des forts niveaux de consensus des praticiens au tour des traitements contre les IST (FC=0,61 soit 
$61 \%)$, les règles douloureuses $(\mathrm{FC}=0,56$ soit $56 \%$ ) et les fibromes utérins ( $\mathrm{FC}=0,34$ soit $34 \%$ ). Selon la littérature, des valeurs de $\mathrm{FC}$ comprises entre $25 \%$ et $73 \%$ sont significatives (Heinrich et al., 1998, Aburjai et al., 2006) ce qui confirme les résultats obtenus par la présente étude. Par ailleurs, les Degrés de fidélité obtenus ont révélé que les quatre espèces aux Facteurs de consensus importants sont en même temps celles de Degrés de fidélité plus élevés (Piper guineense, Capsicum frutescens, Zanthoxylum zanthoxyloides et Aframomum melegueta). Il y a alors de la part des personnes interrogées, une harmonie et une homogénéité du consensus autour des pathologies liées à l'infertilité féminine par rapport aux plantes utilisées pour traiter. De même une fidélité notoire dans l'expression de ce consensus pour les IST a été exprimée. Ces 4 plantes peuvent être susceptibles d'être les plus bioactives parmi les espèces recensées et par conséquent candidates pour des études pharmacologiques. Des travaux ont montrés qu'une plante utilisée par les populations de manière répétitive (avec un DF élevé) pour le traitement d'une pathologie s'avère généralement biologiquement active (Trotter, et al., 1986). D'ailleurs les recherches de bio-activités médicales sont positives à au moins $50 \%$ chez des plantes aux DF et FC intéressants recensées par enquêtes ethnobotaniques (King, et al., 1996). Toutes ces données confirment les résultats auxquels ont abouti les présentes études ethnobotaniques, parce que, la majorité des espèces recensées étant collectées par de récentes études, (MacDonald, et al., 2016, Busia, 2016) ce qui est la prouve que le sujet est d'actualité.

\section{Conclusion}

Les 87 espèces et les 79 recettes utilisées dans la Région sanitaire des Savanes du Togo pour traiter l'infertilité féminine recensées par la présente étude l'ont été avec leurs caractéristiques botaniques et ethnopharmacologiques. Les quatre espèces (Piper guineense, Capsicum frutescens, Zanthoxylum zanthoxyloides et Aframomum melegueta) qui ont été sélectionnées pour de futurs travaux de laboratoire, n'ont pas encore fait l'objet d'études pharmacologiques anti-infertilité féminine. Les présents travaux constituent une première au Togo et ont établi la liste des plantes utilisées dans cette Région sanitaire pour les soins traditionnels à l'infertilité féminine. Les résultats représentent la contribution des auteurs à la valorisation de la médecine traditionnelle du Togo.

\section{Remerciements}

Les auteurs remercient vivement la coordination du CERFOPLAM de l'Université de Lomé (Togo) pour son implication et la Fédération Régionale des Praticiens de la Médecine Traditionnelle des Savanes du Togo pour sa collaboration. 


\section{References:}

1. Aburjai T., Hudaib M., Tayyem R., Yousef M., Qishawi M., (2006). Ethnopharmacological survey of medicinal herbs in Jordan, the Ajloun Heights region. Journal of Ethnopharmacology 110, 294-304.

2. Adjanohoun E.J., Adjakidjè V., Ahyi M.R.A., Ake-Assi L., Akpagana K., Chibon P., Al-Hadji A., Eymé J., Garba M., Gassita J.N., Gbeassor M., Goudote E., Guinko S., Hodouto K.K., Houngnon P., Kéita A., Keoula Y., Klugu-Ocloo W.P., Siamevi K.M., Taffame K.K., (1986). Contribution aux études ethnobotaniques et floristiques au Togo. Agence de Coopération Culturelle et Technique, Paris, 671p.

3. Adjanohoun E.J., Adjakidjè V., Ahyi M.R.A., Ake-Assi L., Akoègninou A., d'Almeida J., Apovo F., Boukef K., Chadare M., Cusset G., Dramane k., Eymé J., Gassita J.N., Gbagidi N., Goudoté E., Guinko S., Houngnon P., Lo I., Saadou M., Sodogandji Th., De Souza S., Tchabi A., Zinsou Dossa C., Zohoun Th., (1989). Contribution aux études ethnobotaniques et floristiques au Bénin. Agence de Coopération Culturelle et Technique, Paris, 895p.

4. Akouègninou A., Van der Burg W.J., Van der Maesen L.J.G, 2006. Flore analytique du Bénin. Backhuys Publishers, 1043p.

5. Alexiades, M.N., (1996). Selected Guidelines for Ethnobotanical Research: A Field Manual. Advances in Economic Botany vol. 10. The New York Botanical Garden, Bronx, 306p.

6. Al-Qarawi A.A., Abdel-Rahman H.A., El-Badry A.A., Harraz F., Razig N.A., Abdel-Magied E.M., 2000.The effect of extracts of Cynomorium coccineum and Withania somnifera on gonadotrophins and ovarian follicles of immature Wistar rats. Phytotherapy Research 14, 288-290.

7. Batawila K., Aménoudji A., Kokou K., de Foucault B., Delelis A., Bouchet P., Akpagana K., (2007). Quelques données ethnobotaniques sur la flore togolaise. Acta Botanica Gallica, 154, 407-422.

8. Busia k., (2016). Fundamentals of Herbal Medicine: Major Plant Families, Analytical Methods, Materia Medica Volume 2, 771p.

9. Cragg M. G, Newman J.D., (2002). Drugs from nature: past achievements, future prospects. Advance in phytomedecine. 01, 23-37.

10. Deleke-Koko, K.I.E., (2005). Utilisation des plantes médicinales contre les maladies et troubles gynécologiques dans les terroirs autour de la Zone Cynégétique de la Pendjari (ZCP) du Bénin : compréhension, inventaire ethnobotanique et perspectives pour leur conservation. Mémoire pour l'obtention du Diplôme d'Ingénieur Agronome, Abomey-Calavi, Bénin. 
11. D.G.S.C.N.T (Direction Générale de la Statistique et de la Comptabilité Nationale) du Togo, (2011). Recensement Général de la Population et de l'Habitat, UNFPA/PNUD, 65p.

12. Dénou A., Koudouvo K., Togola A., Aziati K.Y., Esseh J., Ajavon C.A., Essien K., Aklikokou K., Sanogo R., Diallo D., Gbeassor M., (2016). Traditional knowledge on antimalarial plants having analgesic properties, used in Togo Maritime Region. Journal of Ethnobiology and Traditional Medicine. Photon 126, 1160-1170.

13. Dénou, A., Koudouvo, K., Togola, A., Haïdara, M., Dembélé, S. M., Ballo, F. N., Sanogo, R., Diallo, D., Gbeassor, M., (2016). Savoir traditionnel sur les plantes antipaludiques à propriétés analgésiques, utilisées dans le district de Bamako (Mali). Journal of Applied Biosciences 112: 10985-10995.

14. Eklu-Natey R.D., Balet A., (2012). Pharmacopée africaine. Dictionnaire et monographies multilingues du potentiel médicinal des plantes africaines : Afrique de l'ouest. Volume 1, Lausanne: Éditions d'en-bas; Genève: Traditions et Médecine, 912p.

15. Gazzaneo L., de Lucena R., Albuquerque U., (2005). Knowledge and use of medicinal plants by Local specialists in a region of Atlantic Forest in the state of Permanbuco (Northeastern Brazil). Journal of Ethnopharmacology and Ethnomedicine 1, 1-8.

16. Göhre A., Toto-Nienguesse A.G., Futuro M., Neinhuis C., Lautenschläger T., (2016). Plants from disturbed savannah vegetation and their usage by Bakongo tribes in Uíge, Northern Angola. Journal of Ethnobiology and Ethnomedicine 12:42.

17. Hadj-Seyd A., Kemassi A., Hadj Kouider Y., Harma A., (2016). Traitement de l'infertilité : plantes spontanées du Sahara septentrional. Phytothérapie 14, 241-245.

18. Heinrich M., Ankli A., Frei B., Weimann C., Sticher O., (1998). Medicinal plants in Mexico: Healers consensus and cultural importance. Soc. Sci. Med. vol. 47, $\mathrm{n}^{\circ}$ 11, 1859-1871.

19. Iwu, M.M., (1993). Handbook of African medicinal plants. CRC Press, $257 \mathrm{p}$.

20. Karou S.D., Tchacondo T., Tchibozo M.A.D., Abdoul-Rahaman S., Anani K., Koudouvo K., Batawila K.,Agbonon A., Simpore J., C. de Souza C., (2011). Ethnobotanical study of medicinal plants used in the management of diabetes mellitus and hypertension in the Central Region of Togo. Pharmaceutical Biology 49(12): 1286-97

21. King S.R., Carlson T.J., Moran K., (1996). Biological diversity, indigenous knowledge, drug discovery and intellectual property rights: Creating reciprocity maintaining relationships. Journal of Ethnopharmacology 51(1-3): 45-57. 
22. Koudouvo, K., (2009). Contribution à la recherche sur les plantes médicinales à propriété antipaludique du Togo. Thèse de Doctorat Unique de l'Université de Lomé en Biologie de Développement. Option: Ethnobotanique et Pharmacologie des Substances Naturelles, $182 \mathrm{p}$.

23. Koudouvo K., Karou D.S., Kokou K., Essien K., Aklikokou K., Glitho I.A., Simpore J., Sanogo R., De Souza C., Gbeassor M., (2011). An ethnobotanical study of antimalarial plants in Togo Maritime Region. Journal of Ethnopharmacology 134, 183-190.

24. Koudouvo K., Esseh K., Denou A., Aziati T., Ajavon C., Afanyibo YG., Agbonon A., Sanogo R., Dougnon J., Aklikokou K., Aguiyi J.C., Diallo D., Mensah G.A., Gbeassor M., (2016). Ethnopharmacological study of antimalarial recipes in Togo for a formulation of phytomédecine for malarial taking care. Bulletin de la Recherche Agronomique du Bénin (BRAB) Numéro 79: 1025-2355.

25. K. Koudouvo, A. Denou, K. Asseh, R. Sanogo, K. Essien, D. Diallo, K. Kokou, S. K. Tozo, K. Aklikokou, J. C. Aguiyi and M. Gbéassor (2017). Ethnobotanical Survey of Endangered Antimalarial and Analgesic Plants of Togo for the Safeguard of the Medicinal Biodiversity. Journal of Agriculture and Ecology Research International 12(2) : 1-9

26. Leonti M., Stafford G.I., Dal Cero M., Cabras S., Castellanos M.E., Casu L., Weckerle C.S. (2017). Reverse ethnopharmacology and drug discovery. Journal of Ethnopharmacology, 198, 417-431 doi.org/10.1016/j.jep.2016.12.044

27. Mac Donald, I., Oghale, O-U., Hauwa, G. J., (2016). Ethnomedicinal Survey of Plants used in the Treatment of Female Infertility in Chanchaga Niger State, Nigeria. Marmara Pharmaceutical Journal 20: 269-274.

28. M.S (Ministère de la Santé), République Togolaise, (2015). Rapport annuel et Revue annuelle des activités de la Région des Savanes, 28p.

29. Njoroge N.G., Bussmann W.R., (2007). Ethnotherapeautic management of skin diseases among the Kikuyus of Central Kenya. Journal of Ethnopharmacology 111, 303-307.

30. Noumi E., Amvam Z.P.H., Lontsi D., 1998. Aphrodisiac plants used in Cameroon. Fitoterapia (LXIX) 69, 125-134.

31. Ouatara D., Kouame D., Tiebre M-S., Cisse A., N'Guessan K. E., (2016). Diversité floristique et usages des plantes dans la zone soudanienne du Nord-ouest de la Côte d'Ivoire. Journal of Animal \&Plant Sciences Vol.31, Issue 1: 4815-4830.

32. PAO : Pharmacopée d'Afrique de l'Ouest, 2013. Organisation Ouest Africaine de la Santé (OOAS), Edition $\mathrm{N}^{\circ} 1,257 \mathrm{p}$. Pissang, P., A. 
Agban, P.H. Yao, T. Tchacondo, A.Y. Sadji, S. Effoe, S.D. Karou, and K. Batawila, (2016) Evaluation In Vitro De L'activité Antimicrobienne Des

33. Extraits De Cassia Alata Linn.(Fabaceae). European Scientific Journal 12(21).

34. Soh, N.P., Benoit-Vical, F., (2007). West African plants, a source of future antimalarial drugs. Journal of ethnopharmacology 114, 130-140.

35. Soladoye, M.O.M, Chukwuman, E.C., Sulaiman, , O.M., Feyisola R.T., (2014). Ethnobotanical Survey of Plants Used in the Traditional Treatment of Female Infertility in Southwestern Nigeria. Ethnobotany research and Applications 12, 081-090

36. Teklehaymanot T., Giday M., Midhin G., Mekonn Y., (2007). Knowledge and use of medicinal plants by people around Debre Libanos monastery in Ethiopia. Journal of Ethnopharmacology 111, 271-283.

37. Telefo P.B., Lienou L.L., Yemele M.D., Lemfack M.C., Mouokeu C., Goka C.S., Tagne S.R., Moundipa F.P., (2011). Ethnopharmacological survey of plants used for the treatment of female infertility in Baham, Cameroun. Journal of Ethnopharmacology 136, 178-187

38. Trotter R.T., Logan M.H., (1986).Informants consensus: a new approach for identifying potentially effective medicinal plants. In Plants in Indigenous Medicine and Diet. Edited by: Etkin NL. Redgrave Publishing Company, Bedford Hill, NY; pp: 91-112. 